\title{
HIERARCHICAL CONSTRUCTION OF BOUNDED SOLUTIONS IN CRITICAL REGULARITY SPACES
}

\author{
EITAN TADMOR
}

\begin{abstract}
We construct uniformly bounded solutions for the equations $\operatorname{div} U=f$ and $\operatorname{curl} U=\mathbf{f}$ in the critical cases $f \in L_{\#}^{d}\left(\mathbb{T}^{d}, \mathbb{R}\right)$, and respectively, $\mathbf{f} \in L_{\#}^{3}\left(\mathbb{T}^{3}, \mathbb{R}^{3}\right)$. Criticality in this context, manifests itself by the lack of linear solution operator mapping $L_{\#}^{d} \mapsto L^{\infty}\left(\mathbb{T}^{d}\right)$, [BB03, BB07]. Thus, the intriguing aspect here is that although the problems are linear, the construction of their solution is not.

Our constructions are special cases of a general framework for solving linear equations of the form $\mathscr{L} U=f$, where $\mathscr{L}$ is a linear operator densely defined in Banach space $\mathbb{B}$ with a closed range in a (proper subspace) of Lebesgue space $L_{\#}^{p}(\Omega)$, and with an injective dual $\mathscr{L}^{*}$. The solutions are realized in terms of a multiscale hierarchical representation, $U=\sum_{j=1}^{\infty} \mathbf{u}_{j}$, interesting for its own sake. Here, the $\mathbf{u}_{j}$ 's are constructed recursively as minimizers of $\mathbf{u}_{j+1}=\arg _{\min }\left\{\|\mathbf{u}\|_{\mathbb{B}}+\lambda_{j+1}\left\|r_{j}-\mathscr{L} \mathbf{u}\right\|_{L^{p}(\Omega)}^{p}\right\}$, where $r_{j}:=f-\mathscr{L}\left(\sum_{k=1}^{j} \mathbf{u}_{k}\right)$ are resolved in terms of a dyadic sequence of scales $\lambda_{j+1}:=\lambda_{1} 2^{j}$ with large enough $\lambda_{1} \gtrsim\|f\|_{L^{p}}^{1-p}$. The nonlinear aspect of this construction is a counterpart of the fact that one cannot linearly solve $\mathscr{L} U=f$ in critical spaces.
\end{abstract}

\section{CONTENTS}

1. Introduction and statement of the main results

2. Bounded solutions of $\operatorname{div} U=f \in L_{\#}^{p}(\Omega, \mathbb{R}) \quad 4$

3. Bounded solution of $\operatorname{curl} U=\mathbf{f} \in L_{\#}^{3}\left(\mathbb{T}^{3}, \mathbb{R}^{3}\right) \quad 6$

4. Construction of hierarchical solutions for $\mathscr{L} U=f \in L_{\#}^{p}(\Omega) \quad 6$

4.1. A prototype example: hierarchical solution of $\operatorname{div} U=f \in L_{\#}^{2}\left(\mathbb{T}^{2}\right) \quad 6$

4.2. Hierarchical solutions for $\mathscr{L} U=f \in L_{\#}^{p}(\Omega)$ : approximate solutions 10

4.3. From approximate to exact solutions 13

5. An appendix on $\vee$-minimizers $\quad 15$

References $\quad 17$

"Whenever you can settle a question by explicit construction, be not satisfied with purely existential arguments."

Hermann Weyl, [Weyl46, p. 326]

\section{INTRODUCTION AND STATEMENT OF THE MAIN RESULTS}

We begin with a prototype example for the class of equations alluded to in the title of the paper. Let $L_{\#}^{d}\left(\mathbb{T}^{d}\right)$ denote the Lebesgue space of periodic functions with zero mean over the $d$-dimensional torus $\mathbb{T}^{d}$. Given $f \in L_{\#}^{d}\left(\mathbb{T}^{d}\right)$, we seek a uniformly bounded solution of the problem

$$
\operatorname{div} U=f, \quad U \in L^{\infty}\left(\mathbb{T}^{d}, \mathbb{R}^{d}\right) .
$$

July 6,2014

1991 Mathematics Subject Classification. 35C10, 35F05, 46A30, 49M27, $68 \mathrm{U} 10$.

Key words and phrases. Closed range theorem, hierarchical solutions, duality, Sobolev estimates, divergence, curl, multiscale expansion, image processing, critical regularity.

Research was supported by NSF grants DMS10-08397, RNMS11-07444 (KI-Net) and ONR grant \#N00014-1210318. 
The classical elliptic solution of the first half of (1.1), $U=\nabla \Delta^{-1} f$, lies in $W_{\#}^{1, d}\left(\mathbb{T}^{d}\right)$ which may fail to satisfy the uniform bound sought in the second half of (1.1). Thus, the question is whether (1.1) admits a solution which gains uniform boundedness, $\|U\|_{L^{\infty}} \lesssim\|f\|_{L^{d}}$, at the expense of giving up on the irrotationality condition curl $U=0$. This question was addressed by Bourgain and Brezis, [BB03, Proposition 1]. They prove that (1.1) admits uniformly bounded solutions for all $f \in L_{\#}^{d}\left(\mathbb{T}^{d}\right)$, with the intricate aspect that a solution operator mapping $L_{\#}^{d} \mapsto L^{\infty}$ must be nonlinear; in particular, therefore, the uniform boundedness of irrotational elliptic solution must fail. The existence of such uniformly bounded solutions was proved in [BB03] using a straightforward duality argument based on the closed range theorem.

The purpose of this paper is to present an alternative approach for the existence of such solutions. Our approach is constructive: the solution $U$ is constructed as a hierarchical sum, $U=\sum_{j=1}^{\infty} \mathbf{u}_{j}$, where the $\left\{\mathbf{u}_{j}\right\}$ 's are computed recursively as appropriate minimizers,

$$
\mathbf{u}_{j+1}=\underset{\mathbf{u}}{\arg \min }\left\{\|\mathbf{u}\|_{L^{\infty}}+\lambda_{1} 2^{j}\left\|f-\operatorname{div}\left(\sum_{k=1}^{j} \mathbf{u}_{k}+\mathbf{u}\right)\right\|_{L^{d}}^{d}\right\}, \quad j=0,1, \ldots,
$$

and $\lambda_{1}$ is a sufficiently large parameter specified below. As an example, we refer to [TT11] for the computation of uniformly bounded hierarchical solution of the equation $\operatorname{div} U=\Delta G$ with $G:=$ $x_{1}|\ln r|^{1 / 3} \zeta(r) \in L_{\#}^{2}\left(\mathbb{T}^{2}\right)$ where $\zeta(\cdot)$ is a radial cut-off away from the origin, [BB03, 33 , Remark 7]. The elliptic solution, $U=\nabla G$, has a fractional logarithmic growth at the origin, whereas the computation confirms that the hierarchical solution $U=\sum \mathbf{u}_{j}$ remains uniformly bounded, $\|U\|_{L^{\infty}} \lesssim\|\Delta G\|_{L^{2}}<\infty$.

The above construction is in fact a special case of our main result which applies to general linear problems of the form

$$
\mathscr{L} U=f, \quad f \in L_{\#}^{p}(\Omega), \quad \Omega \subset \mathbb{R}^{d}, 1<p<\infty .
$$

Here, $\mathscr{L}: \mathbb{B} \mapsto L_{\#}^{p}(\Omega)$ is a linear operator densely defined on a Banach space $\mathbb{B}$ with a closed range in $L_{\#}^{p}(\Omega)$. The subscript $\{\cdot\}_{\#}$ indicates an appropriate subspace of $L^{p}$,

$$
L_{\#}^{p}(\Omega)=L^{p}(\Omega) \cap \operatorname{Ker}(\mathscr{P}),
$$

where $\mathscr{P}: L^{p} \mapsto L^{p}$ is a linear operator whose null is "compatible" with the range of $\mathscr{L}$ so that the dual of $\mathscr{L}$ is injective, namely, there exists $\beta>0$ such that

$$
\left\|g-\mathscr{P}^{*} g\right\|_{L^{p^{\prime}}} \leq \beta\left\|\mathscr{L}^{*} g\right\|_{\mathbb{B}^{*}}, \quad \forall g \in L^{p^{\prime}}(\Omega) .
$$

The closed range theorem combined with the open mapping principle tell us that if the a priori duality estimate assumed in (1.3) holds, then equation (1.2) admits a solution, $\|U\|_{\mathbb{B}} \lesssim \gamma\|f\|_{L^{p}}$ with a constant $\gamma=\gamma(\beta, p, d)$. Our main result explains the existence of such $U$ s by explicit construction.

Theorem 1.1. Fix $1<p<\infty$ and assume that the a priori estimate (1.3) holds. There exists $\gamma<\infty$ (depending on $p$ and linearly on $\beta$ ) such that for any given $f \in L_{\#}^{p}(\Omega)$, the equation $\mathscr{L} U=f$ admits a hierarchical solution of the form $U=\sum_{j=1}^{\infty} \mathbf{u}_{j} \in \mathbb{B}$,

$$
\|U\|_{\mathbb{B}} \leq \gamma\|f\|_{L^{p}}, \quad \gamma<\infty .
$$

Here, the $\left\{\mathbf{u}_{j}\right\}$ 's are constructed recursively as minimizers of

$$
\mathbf{u}_{j+1}=\underset{\mathbf{u}}{\arg \min }\left\{\|\mathbf{u}\|_{\mathbb{B}}+\lambda_{j+1}\left\|f-\mathscr{L}\left(\sum_{k=1}^{j} \mathbf{u}_{k}+\mathbf{u}\right)\right\|_{L^{p}}^{p}\right\}, \quad j=0,1, \ldots,
$$

where $\left\{\lambda_{j}\right\}_{j \geq 1}$ is a dyadic sequence, $\lambda_{j+1}:=\lambda_{1} 2^{j}$ with a sufficiency large $\lambda_{1}>\frac{\beta}{p}\|f\|_{L^{p}}^{1-p}$. 
Remark 1.1 (Exponential convergence). The description of $U$ as the sum $U=\sum \mathbf{u}_{j}$ provides a multiscale hierarchical decomposition of a solution for (1.2) for rapidly increasing sequence of scales, $\lambda_{j+1}=\lambda_{1} \zeta^{j}$ with any $\zeta>1$. The role $\left\{\lambda_{j}\right\}$ 's as the different scales associated with the $\mathbf{u}_{j}$ 's is reflected through the exponential decay bound (consult (4.23) below)

$$
\left\|\mathbf{u}_{j}\right\|_{\mathbb{B}} \lesssim \frac{\lambda_{j+1}}{\lambda_{j}^{p^{\prime}}} \sim\|f\|_{L_{\#}^{p}} \zeta^{-\frac{j}{p-1}}, \quad \lambda_{j+1}=\lambda_{1} \zeta^{j} \sim \frac{\zeta^{j}}{\|f\|_{L_{\#}^{p-1}}^{p}}, \quad 1<p<\infty .
$$

For simplicity, we limit our discussion to the dyadic case $\zeta=2$.

Remark 1.2 (On the a priori duality estimate (1.3)). The a priori estimate (1.3) is exactly what is needed for the hierarchical construction $\sum \mathbf{u}_{j}$ to converge. It should be emphasized, however, that the construction does not require knowledge of the precise value of the constant $\beta$ appearing in estimate (1.3). Indeed, the parameter $\beta$ enters through the initial scale, $\lambda_{1}$, which is to be chosen large enough,

$$
\lambda_{1} \geq \frac{\beta}{p}\|f\|_{L^{p}}^{1-p},
$$

so that by lemma 5.3, it dictates a non-trivial first hierarchical step,

$$
\mathbf{u}_{1}=\underset{\mathbf{u}}{\arg \min }\left\{\|\mathbf{u}\|_{\mathbb{B}}+\lambda_{1}\|f-\mathscr{L} \mathbf{u}\|_{L^{p}}^{p}\right\} .
$$

What happens if the initial scale $\lambda_{1}$ is underestimated relative to an unknown value of $\beta$ ? then, as noted in lemma 5.2 below, the variational statement (1.5) will yield zero hierarchical terms, $\mathbf{u}_{j} \equiv 0$ for increasing sequence of scales $\lambda_{1} 2^{j}, j=1,2, \ldots$, until reaching the critical scale such that $p \lambda_{1} 2^{j_{0}} \gtrsim$ $\beta\|f\|_{L^{p}}^{1-p}$, which will dictate the first non-trivial step of the hierarchical decomposition, $U=\sum_{j=j_{0}} \mathbf{u}_{j}$. In this sense, the construction of hierarchical solution, $U=\sum \mathbf{u}_{j}$ is independent of the precise value of $\beta$ in (1.3): the latter is only needed to guarantee that the hierarchical construction will indeed pick up the first non-trivial minimizer after finitely many steps $j_{0}$.

Remark 1.3 (The limiting cases $p=1, \infty)$. The $L^{p}$-valued hierarchical constructions in theorem 1.1 can be extended to a more general setup of operators valued in Orlicz spaces (outlined in remark 4.4 below). The limiting cases, however, are excluded; for example, there exist no $\dot{W}^{1, p}$ solutions of $\operatorname{div} U=f$ for general $f \in L^{p}$ with $p=1, \infty$, [BB03, Section 2], [DFT05]. The iterative aspect of the hierarchical construction is reminiscent of Artola \& Tartar construction of $L^{p}(\mathbb{R})$-functions for the end case $p=1$, as a limiting case for interpolation of $W^{1,1}\left(\mathbb{R}^{2}\right)$-traces, [Tar94, §II],[Ga57].

$L^{2}$-based hierarchical decompositions were introduced by us in the context of image processing, [TNV04, TNV08], and motivated the present construction of solutions in the more general setup of the closed range theorem. We demonstrate such hierarchical constructions of solution to two important examples of critical regularity studied by Bourgain \& Brezis, [BB03, BB07]. These are the construction of uniformly bounded solutions to $\operatorname{div} U=f \in L_{\#}^{d}\left(\mathbb{T}^{d}\right)$, discussed in section 2 , and construction of uniformly bounded solutions to $\operatorname{curl} U=\mathbf{f} \in L_{\#}^{3}\left(\mathbb{T}^{3}, \mathbb{R}^{3}\right)$ discussed in section 3 . The critical regularity in these cases manifests itself in terms of lack of right inverses for $\mathscr{L}$ bounded on the corresponding critical $L^{p}$ spaces, or equivalently, $\operatorname{Ker} \mathscr{L}$ which cannot be complemented in $L^{\infty}$, [BB03, §3],[Aji10, §5.3].

The main novelty of theorem 1.1 is using these hierarchical decompositions for explicit construction of solutions for general equations governed by operators with a closed range in $L_{\#}^{p}, 1<p<\infty$. The proof of the special case $p=2$ is given in section 4.1: here, we trace precise bounds and clarify their role in the hierarchical construction. The $L^{2}$-case serves as the prototype case for the general 
setup of hierarchical constructions in $L^{p}$ spaces in section 4. Finally, the characterization of minimizers, such as those encountered in (1.5), is summarized in section 5 .

Acknowledgment. I indebted to Haim Brezis for discussions on the works [BB03, BB07], to François Golse, Giuseppe Savaré and Terence Tao who, respectively, brought to my attention [Jam47], [Ga57, Tar94] and [Aji10], and to Przemyslaw Wojtaszczyk for his comments on an earlier version of this paper [Woj10].

\section{Bounded SOLutions of $\operatorname{div} U=f \in L_{\#}^{p}(\Omega, \mathbb{R})$}

Let $\mathscr{P}$ denote the averaging projection, $\mathscr{P} g:=\bar{g}$ where $\bar{g}$ is the average value of $g$. Given $f \in L_{\#}^{p}\left(\mathbb{T}^{d}\right):=\left\{g \in L^{p}\left(\mathbb{T}^{d}\right) \mid \bar{g}=0\right\}$, then according to theorem 1.1, we can construct hierarchical solutions of

$$
\operatorname{div} U=f, \quad f \in L_{\#}^{p}\left(\mathbb{T}^{d}\right), \quad 1<p<\infty,
$$

in an appropriate Banach space, $U \in \mathbb{B}$, provided the corresponding a priori estimate (1.3) holds, namely, there exists a constant $\beta>0$ (which may vary of course, depending on $p, d$ and $\mathbb{B}$ ), such that

$$
\|g-\bar{g}\|_{L^{p^{\prime}}} \leq \beta\|\nabla g\|_{\mathbb{B}^{*}}, \quad \forall g \in L^{p^{\prime}}\left(\mathbb{T}^{d}\right) .
$$

We specify four cases of such relevant $\mathbb{B}$ 's.

\#1. Solution of $\operatorname{div} U=f \in L_{\#}^{p}$ with $U \in \dot{W}^{1, p}$. Since

$$
\|g-\bar{g}\|_{L^{p^{\prime}}\left(\mathbb{T}^{d}\right)} \leq\|\nabla g\|_{\dot{W}^{-1, p^{\prime}}\left(\mathbb{T}^{d}, \mathbb{R}^{d}\right)}, \quad \forall g \in L^{p^{\prime}}\left(\mathbb{T}^{d}\right),
$$

we can construct hierarchical solutions of (2.1) in $\mathbb{B}=\dot{W}^{1, p}\left(\mathbb{T}^{d}, \mathbb{R}^{d}\right), 1<p<\infty$. This is the same integrability space of the irrotational solution of (2.1), $\nabla \Delta^{-1} f \in \dot{W}^{1, p}\left(\mathbb{T}^{d}, \mathbb{R}^{d}\right)$.

\#2. Solution of $\operatorname{div} U=f \in L_{\#}^{p}$ with $U \in L^{p^{*}}$. By Sobolev inequality

$$
\|g-\bar{g}\|_{L^{p^{\prime}\left(\mathbb{T}^{d}\right)}} \leq \beta\|\nabla g\|_{L^{\left(p^{*}\right)^{\prime}\left(\mathbb{T}^{d}, \mathbb{R}^{d}\right)}}, \quad \frac{1}{p^{*}}=\frac{1}{p}-\frac{1}{d}, \quad d \leq p<\infty, \quad \forall g \in L^{p^{\prime}}\left(\mathbb{T}^{d}\right),
$$

where the case $p=d$ corresponds to the isoperimetric Gagliardo-Nirenberg inequality, [DPD02, CNV04] $\|g-\bar{g}\|_{L^{d^{\prime}\left(\mathbb{T}^{d}\right)}} \leq \beta\|g\|_{\dot{W}^{1,1}\left(\mathbb{T}^{d}\right)}$. We distinguish between two cases.

(i) The case $d<p<\infty$ : the equation $\operatorname{div} U=f \in L_{\#}^{p}\left(\mathbb{T}^{d}\right)$ has a solution $U \in L^{p^{*}}\left(\mathbb{T}^{d}, \mathbb{R}^{d}\right)$. This is the same integrability space of the irrotational solution $\nabla \Delta^{-1} f \in W^{1, p}\left(\mathbb{T}^{d}, \mathbb{R}^{d}\right) \subset L^{p^{*}}\left(\mathbb{T}^{d}, \mathbb{R}^{d}\right)$.

(ii) The case $d=p$ : the equation $\operatorname{div} U=f \in L_{\#}^{d}\left(\mathbb{T}^{d}\right)$ has a solution $U \in L^{\infty}\left(\mathbb{T}^{d}, \mathbb{R}^{d}\right)$. This is the the prototype example discussed in the beginning of the introduction. According to the intriguing observation of Bourgain \& Brezis, [BB03, Proposition 2], there exists no bounded right inverse $K$ : $L_{\#}^{d} \mapsto L^{\infty}$ for the operator div, and therefore, there exists no linear construction of solutions $f \mapsto U$ (in particular, $\nabla \Delta^{-1} f$ cannot be uniformly bounded). Theorem 1.1 provides a nonlinear hierarchical construction of such solutions. The computation of such $L^{\infty}$-solutions using hierarchical iterations in the two-dimensional critical case was carried out in [TT11].

Remark 2.1 (Homogeneity). We rewrite the hierarchical iteration (1.5) with $\lambda_{1}=C\|f\|_{L^{p}}^{1-p}$ in the form

$$
\left[\mathbf{u}_{j+1}, r_{j+1}\right]=\underset{\mathscr{L} \mathbf{u}+r=r_{j}}{\operatorname{argmin}}\left\{\|\mathbf{u}\|_{\mathbb{B}}+C 2^{j} \frac{\|r\|_{L^{p}}^{p}}{\|f\|_{L^{p}}^{p-1}}\right\}, \quad r_{j}:= \begin{cases}f, & j=0 \\ f-\mathscr{L}\left(\sum_{k=1}^{j} \mathbf{u}_{k}\right), & j=1,2 \ldots\end{cases}
$$

Observe that if $\left[\mathbf{u}_{1}, r_{1}\right]$ is the first minimizer associated with $r_{0}=f$, then the corresponding first minimizer associated with $\alpha f$ is $\left[\alpha \mathbf{u}_{1}, \alpha r_{1}\right]$, and recursively, the next hierarchical components are 
$\left[\alpha \mathbf{u}_{j}, \alpha r_{j}\right]$. Thus, as noted in [TNV08, remark 1.1], the hierarchical solution is homogeneous of degree one: if $U=U_{f}$ specifies the (nonlinear) dependence of hierarchical solution on $f$, then $U_{\{\alpha f\}}=$ $\alpha U_{f}$.

\#3. Solution of $\operatorname{div} U=f \in L_{\#}^{d}$ with $U \in L^{\infty} \cap \dot{W}^{1, d}$. A central question raised and answered in [BB03] is whether (2.1) has a solution which captures the joint regularity, $U \in \mathbb{B}=L^{\infty} \cap \dot{W}^{1, d}\left(\mathbb{T}^{d}, \mathbb{R}^{d}\right)$. To this end, one needs to verify the duality estimate (2.2), which now reads

$$
\|g-\bar{g}\|_{L^{d^{\prime}\left(\mathbb{T}^{d}\right)}} \leq \beta\|\nabla g\|_{L^{1}+\dot{W}^{-1, d^{\prime}}\left(\mathbb{T}^{d}, \mathbb{R}^{d}\right)}, \quad \forall g \in L^{d^{\prime}}\left(\mathbb{T}^{d}\right) .
$$

This key estimate was proved in [BB03]. Thus, theorem 1.1 converts (2.4) into a constructive proof of:

Corollary 2.1. The equation $\operatorname{div} U=f \in L_{\#}^{d}\left(\mathbb{T}^{d}\right)$ admits a solution $U \in L^{\infty} \cap \dot{W}^{1, d}\left(\mathbb{T}^{d}, \mathbb{R}^{d}\right)$, given by the hierarchical decomposition $U=\sum_{j=1} \mathbf{u}_{j}$, which is constructed by the refinement step,

$$
\mathbf{u}_{j+1}=\underset{\mathbf{u}}{\arg \min }\left\{\|\mathbf{u}\|_{L^{\infty} \cap \dot{W}^{1, d}}+\lambda_{1} 2^{j}\left\|f-\operatorname{div}\left(\sum_{k=1}^{j} \mathbf{u}_{k}+\mathbf{u}\right)\right\|_{L^{d}}^{d}\right\}, \quad j=0,1,2 \ldots,
$$

with sufficiently large $\lambda_{1} \geq \frac{\beta}{d}\|f\|_{L^{d}}^{1-d}$.

Remark 2.2. We comment here the key role of the duality estimate (2.4). The case $d=2$ was proved by a direct method outlined in [BB03, Section 4]; alternative two-dimensional proofs were given by Maz'ya [Ma07] and Mironescu [Mi10]. For $d>2$, however, the derivation of (2.4) was proved in Bourgain \& Brezis [BB03, theorem 1] as a byproduct of their construction of $L^{\infty} \cap \dot{W}^{1, d}$ solutions for $\operatorname{div} U=f$ !. The construction, based on an intricate Littlewood-Paley decomposition is rather involved [BB03, section 6], and to our knowledge, a simpler, direct derivation of (2.4) is still open. Thus, corollary 2.1 - which still depends on the construction of Bourgain \& Brezis to justify (2.4), offers a simpler alternative for the construction of such $L^{\infty} \cap \dot{W}^{1, d}$-bounded solutions in terms of the minimizers,

$$
\vee_{\text {div }}(r, \lambda):=\inf _{\mathbf{u}}\left\{\|\mathbf{u}\|_{L^{\infty} \cap \dot{W}^{1, d}}+\lambda\|r-\operatorname{div} \mathbf{u}\|_{L^{d}}^{d}\right\}
$$

Computation of the related $L^{\infty}$-based minimizers were carried out in [GLMV07, LV05] and it would be desirable to develop efficient algorithms to compute the corresponding minimizers of $\vee_{\text {div }}\left(r, \lambda ; L^{\infty} \cap\right.$ $\left.\dot{W}^{1, d}\right)$. Spectral approximation of such minimizers was discussed in [Ma06].

Since the proof of the dual estimate (2.4) in $d>2$ dimensions is indirect, a specific value of $\beta$ is not known. As noted in remark 1.2, however, the hierarchical construction can proceed without a priori knowledge of the precise value of $\beta$ : if one sets $\lambda_{1}=\|f\|_{L^{d}}^{1-d}$ and this initial scale underestimates a correct value of, say, $\beta>1$, then it will take at most $j_{0} \sim \log (\beta)$ steps before picking-up non-trivial terms in the hierarchical decomposition, $U=\sum_{j=j_{0}} \mathbf{u}_{j}$.

\#4. Solution of $\operatorname{div} U=f \in L_{\#}^{d}(\Omega)$ with $U \in L^{\infty} \cap \dot{W}_{0}^{1, d}(\Omega)$. The constructions of bounded solutions for (2.1) extend to the case of Lipschitz domains, $\Omega \subset \mathbb{R}^{d}$, see [BB03, section 7.2]. For future reference we state the following.

Corollary 2.2. Given $f \in L_{\#}^{d}(\Omega):=\left\{g \in L^{d}(\Omega) \mid \int_{\Omega} g(x) d x=0\right\}$, then the equation div $U=f$ admits a solution $U \in L^{\infty} \cap \dot{W}_{0}^{1, d}\left(\Omega, \mathbb{R}^{d}\right)$, such that

$$
\|U\|_{L^{\infty} \cap \dot{W}^{1, d}(\Omega)} \leq \gamma\|f\|_{L^{d}(\Omega)} .
$$


It is given by the hierarchical decomposition, $U=\sum_{j=1} \mathbf{u}_{j}$, which is constructed by the refinement step,

$$
\mathbf{u}_{j+1}=\underset{\mathbf{u}: \mathbf{u}_{\mid \partial \Omega}=0}{\arg \min }\left\{\|\mathbf{u}\|_{L^{\infty} \cap \dot{W}^{1, d}(\Omega)}+\lambda_{1} 2^{j}\left\|f-\operatorname{div}\left(\sum_{k=1}^{j} \mathbf{u}_{k}+\mathbf{u}\right)\right\|_{L^{d}(\Omega)}^{d}\right\}, j=0,1,2 \ldots,
$$

with sufficiently large $\lambda_{1} \gtrsim \beta\|f\|_{L^{d}(\Omega)}^{1-d}$.

\section{Bounded SOLUTION OF curl $U=\mathbf{f} \in L_{\#}^{3}\left(\mathbb{T}^{3}, \mathbb{R}^{3}\right)$}

Let $L_{\#}^{3}\left(\mathbb{T}^{3}, \mathbb{R}^{3}\right)$ denote the $L^{3}$-subspace of divergence-free 3 -vectors with zero mean. We seek solutions of

$$
\operatorname{curl} U=\mathbf{f}, \quad \mathbf{f} \in L_{\#}^{3}\left(\mathbb{T}^{3}, \mathbb{R}^{3}\right),
$$

in an appropriate Banach space, $U \in \mathbb{B}$. We appeal to the framework of hierarchical solutions in theorem 1.1, where $\mathscr{P}: L^{3}\left(\mathbb{T}^{3}, \mathbb{R}^{3}\right) \mapsto L^{3}\left(\mathbb{T}^{3}, \mathbb{R}^{3}\right)$ is the irrotational portion of Hodge decomposition with a dual, $\mathscr{P}^{*} \mathbf{g}:=\nabla \Delta^{-1} \operatorname{div} \mathbf{g}-\overline{\mathbf{g}}$. According to theorem 1.1, we can construct hierarchical solutions, $U \in \mathbb{B}$ of (3.1), provided (1.3) holds

$$
\left\|\mathbf{g}-\mathscr{P}^{*} \mathbf{g}\right\|_{L^{3 / 2}} \leq \beta \| \text { curl } \mathbf{g} \|_{\mathbb{B}^{*}}, \quad \mathbf{g} \in L^{3 / 2}\left(\mathbb{T}^{3}, \mathbb{R}^{3}\right) .
$$

Since $\left\|\mathbf{g}-\mathscr{P}^{*} \mathbf{g}\right\|_{L^{3 / 2}} \lesssim \|$ curl $\mathbf{g} \|_{\dot{W}^{-1,3 / 2}}$, we can construct hierarchical solutions of (3.1) in $\dot{W}^{1,3}$. This has the same integrability as the divergence-free solution of $(3.1),(-\Delta)^{-1} \mathrm{curl} \mathbf{f}$. A more intricate question is whether (3.1) admits a uniformly bounded solution, since such a solution cannot be constructed by a linear procedure. These solutions were constructed by Bourgain and Brezis in [BB07, Corollary 8'], which in turn imply the key a priori estimate,

$$
\left\|\mathbf{g}-\mathscr{P}^{*} \mathbf{g}\right\|_{L^{3 / 2}\left(\mathbb{T}^{3}, \mathbb{R}^{3}\right)} \leq \beta\|\operatorname{curl} \mathbf{g}\|_{L^{1}+\dot{W}^{-1,3 / 2}\left(\mathbb{T}^{3}, \mathbb{R}^{3}\right)}, \quad \forall \mathbf{g} \in L^{3 / 2}\left(\mathbb{T}^{3}, \mathbb{R}^{3}\right): \operatorname{div} \mathbf{g}=\overline{\mathbf{g}}=0
$$

Granted (3.3), theorem 1.1 offers a simpler alternative to the construction in [BB07] based on the following hierarchical decomposition.

Corollary 3.1. The equation curl $U=\mathbf{f} \in L_{\#}^{3}\left(\mathbb{T}^{3}, \mathbb{R}^{3}\right)$, admits a solution $U \in L^{\infty} \cap \dot{W}^{1,3}\left(\mathbb{T}^{3}, \mathbb{R}^{3}\right)$,

$$
\|U\|_{L^{\infty} \cap \dot{W}^{1,3}\left(\mathbb{T}^{3}, \mathbb{R}^{3}\right)} \leq \gamma\|\mathbf{f}\|_{L^{3}\left(\mathbb{T}^{3}, \mathbb{R}^{3}\right)},
$$

which can be constructed by the (nonlinear) hierarchical expansion, $U=\sum \mathbf{u}_{j}$,

$$
\mathbf{u}_{j+1}=\underset{\mathbf{u}}{\arg \min }\left\{\|\mathbf{u}\|_{L^{\infty} \cap \dot{W}^{1,3}}+\lambda_{1} 2^{j}\left\|\mathbf{f}-\operatorname{curl}\left(\sum_{k=1}^{j} \mathbf{u}_{k}+\mathbf{u}\right)\right\|_{L^{3}\left(\mathbb{T}^{3}, \mathbb{R}^{3}\right)}^{3}\right\}, \quad j=0,1, \ldots,
$$

with sufficiently large $\lambda_{1} \geq \frac{\beta}{3}\|\mathbf{f}\|_{L^{3}\left(\mathbb{T}^{3}, \mathbb{R}^{3}\right)}^{-2}$.

4. Construction of hierarchical solutions for $\mathscr{L} U=f \in L_{\#}^{p}(\Omega)$

4.1. A prototype example: hierarchical solution of $\operatorname{div} U=f \in L_{\#}^{2}\left(\mathbb{T}^{2}\right)$. We begin our discussion on hierarchical constrictions with a two-dimensional prototype example of

$$
\operatorname{div} U=f, \quad f \in L_{\#}^{2}\left(\mathbb{T}^{2}\right):=\left\{g \in L^{2}\left(\mathbb{T}^{2}\right) \mid \int_{\mathbb{T}^{2}} g(x) d x=0\right\} .
$$

Our starting point for the construction of a uniformly bounded solution of (4.1) is a decomposition of $f$,

$$
f=\operatorname{div} \mathbf{u}_{1}+r_{1}, \quad f \in L_{\#}^{2}\left(\mathbb{T}^{2}\right)
$$


where $\left[\mathbf{u}_{1}, r_{1}\right]$ is a minimizing pair of the functional,

$$
\left[\mathbf{u}_{1}, r_{1}\right]=\underset{\operatorname{div} \mathbf{u}+r=f}{\arg \min }\left\{\|\mathbf{u}\|_{L^{\infty}}+\lambda_{1}\|r\|_{L^{2}}^{2}\right\} .
$$

Here $\lambda_{1}$ is a fixed parameter at our disposal: if we choose $\lambda_{1}$ large enough, $\lambda_{1}>\frac{1}{2\|f\|_{\dot{W}^{1,1}}}$, then according to lemma 5.3 below, (4.2b) admits a minimizer, $\left[\mathbf{u}_{1}, r_{1}\right]$, satisfying,

$$
\left\|r_{1}\right\|_{\dot{W}^{1,1}}=\frac{1}{2 \lambda_{1}}
$$

To proceed we invoke the isoperimetric Gagliardo-Nirenberg inequality, which states that there exists $\beta>0$ (any $\beta \geq 1 / \sqrt{4 \pi}$ will do), such that for all bounded variation $g$ 's with zero mean,

$$
\|g\|_{L^{2}} \leq \beta\|g\|_{\dot{W}^{1,1}}, \quad \int_{\mathbb{T}^{2}} g(x) d x=0
$$

Since $f$ has a zero mean so does the residual $r_{1}$ and (4.3) yields

$$
\left\|r_{1}\right\|_{L^{2}} \leq \beta\left\|r_{1}\right\|_{\dot{W}^{1,1}}=\frac{\beta}{2 \lambda_{1}}
$$

We conclude that the residual $r_{1} \in L_{\#}^{2}\left(\mathbb{T}^{2}\right)$, and we can therefore implement the same variational decomposition of $f$ in (4.2), and use it to decompose $r_{1}$ with scale $\lambda=\lambda_{2}>\lambda_{1}=\frac{1}{2\left\|r_{1}\right\|_{\dot{W}^{111}}}$. This yields

$$
r_{1}=\operatorname{div} \mathbf{u}_{2}+r_{2}, \quad\left[\mathbf{u}_{2}, r_{2}\right]=\underset{\operatorname{div} \mathbf{u}+r=r_{1}}{\operatorname{argmin}}\left\{\|\mathbf{u}\|_{L^{\infty}}+\lambda_{2}\|r\|_{L^{2}}^{2}\right\} .
$$

Combining this with (4.2a) we obtain $f=\operatorname{div} U_{2}+r_{2}$, where $U_{2}:=\mathbf{u}_{1}+\mathbf{u}_{2}$ is viewed as an improved approximate solution of (4.1) in the sense that it has a smaller residual, $r_{2}$,

$$
\left\|r_{2}\right\|_{L^{2}} \leq \beta\left\|r_{2}\right\|_{\dot{W}^{1,1}}=\frac{\beta}{2 \lambda_{2}}
$$

when compared with the previous residual $\beta\left\|r_{1}\right\|_{B V}=\frac{\beta}{2 \lambda_{1}}$. This process can be repeated: if $r_{j} \in$ $L_{\#}^{2}\left(\mathbb{T}^{2}\right)$ is the residual at step $j$, then we decompose it

$$
r_{j}=\operatorname{div} \mathbf{u}_{j+1}+r_{j+1},
$$

where $\left[\mathbf{u}_{j+1}, r_{j+1}\right]$ is a minimizing pair of

$$
\left[\mathbf{u}_{j+1}, r_{j+1}\right]=\underset{\operatorname{div} \mathbf{u}+r=r_{j}}{\arg \min }\left\{\|\mathbf{u}\|_{L^{\infty}}+\lambda_{j+1}\|r\|_{L^{2}}^{2}\right\}, \quad j=0,1, \ldots
$$

For $j=0$, the decomposition (4.4) is interpreted as (4.2a) by setting $r_{0}:=f$. Note that the recursive decomposition (4.4a) depends on the invariance of $r_{j} \in L_{\#}^{2}\left(\mathbb{T}^{2}\right)$ : if $r_{j}$ has a zero mean then so does $r_{j+1}$, and by (4.3) $r_{j+1} \in L_{\#}^{2}\left(\mathbb{T}^{2}\right)$. The iterative process depends on a sequence of increasing scales, $\lambda_{1}<\lambda_{2}<\ldots \lambda_{j+1}$, which are yet to be determined.

The telescoping sum of the first $k$ steps in (4.4a) yields an improved approximate solution, $U_{k}:=$ $\sum_{j=1}^{k} \mathbf{u}_{j}$ :

$$
f=\operatorname{div} U_{k}+r_{k}, \quad\left\|r_{k}\right\|_{L^{2}} \leq \beta\left\|r_{k}\right\|_{\dot{W}^{1,1}}=\frac{\beta}{2 \lambda_{k}} \downarrow 0, \quad k=1,2, \ldots
$$


The key question is whether the $U_{k}$ 's remain uniformly bounded, and it is here that we use the freedom in choosing the scaling parameters $\lambda_{k}$ : comparing the minimizing pair $\left[\mathbf{u}_{j+1}, r_{j+1}\right]$ of (4.4b) with the trivial pair $\left[\mathbf{u} \equiv 0, r_{j}\right]$ implies, in view of (4.5),

$$
\left\|\mathbf{u}_{j+1}\right\|_{L^{\infty}}+\lambda_{j+1}\left\|r_{j+1}\right\|_{L^{2}}^{2} \leq \lambda_{j+1}\left\|r_{j}\right\|_{L^{2}}^{2} \leq \begin{cases}\lambda_{1}\|f\|_{L^{2}}^{2}, & j=0, \\ \frac{\beta^{2} \lambda_{j+1}}{4 \lambda_{j}^{2}}, & j=1,2, \ldots\end{cases}
$$

We conclude that by choosing a sufficiently fast increasing $\lambda_{j}$ 's such that $\sum_{j} \lambda_{j+1} \lambda_{j}^{-2}<\infty$, then the approximate solutions $U_{k}=\sum_{1}^{k} \mathbf{u}_{j}$ form a Cauchy sequence in $L^{\infty}$ whose limit, $U=\sum_{1}^{\infty} \mathbf{u}_{j}$, satisfies the following.

Theorem 4.1. Fix $\beta$ such that (4.3) holds. Then, for any given $f \in L_{\#}^{2}\left(\mathbb{T}^{2}\right)$, there exists a uniformly bounded solution of (4.1),

$$
\operatorname{div} U=f, \quad\|U\|_{L^{\infty}} \leq 2 \beta\|f\|_{L^{2}} .
$$

The solution $U$ is given by $U=\sum_{j=1}^{\infty} \mathbf{u}_{j}$, where the $\left\{\mathbf{u}_{j}\right\}$ 's are constructed recursively as minimizers of

$$
\left[\mathbf{u}_{j+1}, r_{j+1}\right]=\underset{\operatorname{div} \mathbf{u}+r=r_{j}}{\arg \min }\left\{\|\mathbf{u}\|_{L^{\infty}}+\lambda_{1} 2^{j}\|r\|_{L^{2}}^{2}\right\}, \quad r_{0}:=f, \quad \lambda_{1}=\frac{\beta}{\|f\|_{L^{2}}} .
$$

Proof. With $\lambda_{j}=\lambda_{1} 2^{j-1}$ we have $\left\|U_{k}-U_{\ell}\right\|_{L^{\infty}} \lesssim \sum \lambda_{j+1} \lambda_{j}^{-2} \lesssim 2^{-\ell}, k>\ell \gg 1$. Let $U$ be the limit of the Cauchy sequence $\left\{U_{k}\right\}$ then $\left\|U_{j}-U\right\|_{L^{\infty}}+\left\|\operatorname{div} U_{j}-f\right\|_{L^{2}} \lesssim 2^{-j} \rightarrow 0$, and since $d i v$ has a closed graph on its domain $D:=\left\{\mathbf{u} \in L^{\infty}: \operatorname{div} \mathbf{u} \in L^{2}\left(\mathbb{T}^{2}\right)\right\}$, it follows that $\operatorname{div} U=f$. By (4.6) we have

$$
\|U\|_{L^{\infty}} \leq \sum_{j=1}^{\infty}\left\|\mathbf{u}_{j}\right\|_{L^{\infty}} \leq \lambda_{1}\|f\|_{L^{2}}^{2}+\frac{\beta^{2}}{4 \lambda_{1}} \sum_{j=2}^{\infty} \frac{1}{2^{j-3}}=\lambda_{1}\|f\|_{L^{2}}^{2}+\frac{\beta^{2}}{\lambda_{1}} .
$$

Here $\lambda_{1}>\frac{1}{2\|f\|_{\dot{W}^{1,1}}}$ is a free parameter at our disposal: we choose $\lambda_{1}:=\beta /\|f\|_{L^{2}}$ which by (4.3) is admissible, $\lambda_{1}=\frac{\beta}{\|f\|_{L^{2}}}>\frac{1}{2\|f\|_{\dot{W}^{1,1}}}$, and (4.7) follows.

Remark 4.1 (Energy decomposition). A telescoping summation of the left inequality of (4.6) yields

$$
\sum_{j=1}^{\infty} \frac{1}{\lambda_{j}}\left\|\mathbf{u}_{j}\right\|_{L^{\infty}} \leq\|f\|_{L^{2}}^{2}
$$

setting $\lambda_{j}=\frac{\beta 2^{j-1}}{2\|f\|_{L^{2}}}$ we conclude the "energy bound"

$$
\sum_{j=1}^{\infty} \frac{1}{2^{j-1}}\left\|\mathbf{u}_{j}\right\|_{L^{\infty}} \leq \frac{\beta}{2}\|f\|_{L^{2}}
$$

In fact, a precise energy equality can be formulated in this case, using the characterization of the minimizing pair (consult theorem 5.1 below), $2\left(r_{j+1}, \operatorname{div} \mathbf{u}_{j+1}\right)=\left\|\mathbf{u}_{j+1}\right\|_{L^{\infty}} / \lambda_{j+1}$ : by squaring the refinement step $r_{j}=r_{j+1}+\operatorname{div} \mathbf{u}_{j+1}$ we find

$$
\left\|r_{j}\right\|_{L^{2}}^{2}-\left\|r_{j+1}\right\|_{L^{2}}^{2}=2\left(r_{j+1}, \operatorname{div} \mathbf{u}_{j+1}\right)+\left\|\operatorname{div} \mathbf{u}_{j+1}\right\|_{L^{2}}^{2}=\frac{1}{\lambda_{j+1}}\left\|\mathbf{u}_{j+1}\right\|_{L^{\infty}}+\left\|\operatorname{div} \mathbf{u}_{j+1}\right\|_{L^{2}}^{2} .
$$


A telescoping sum of the last equality yields

Corollary 4.2. Let $U=\sum_{j=1}^{\infty} \mathbf{u}_{j} \in L^{\infty}$ be a hierarchical solution of div $U=f, f \in L_{\#}^{2}\left(\mathbb{T}^{2}\right)$. Then

$$
\frac{1}{\lambda_{1}} \sum_{j=1}^{\infty} \frac{1}{2^{j-1}}\left\|\mathbf{u}_{j}\right\|_{L^{\infty}}+\sum_{j=1}^{\infty}\left\|\operatorname{div} \mathbf{u}_{j}\right\|_{L_{\#}^{2}\left(\mathbb{T}^{2}\right)}^{2}=\|f\|_{L_{\#}^{2}\left(\mathbb{T}^{2}\right)}^{2}, \quad \lambda_{1}=\frac{\beta}{2\|f\|_{L^{2}}}
$$

We mention two examples related to the two-dimensional setup of theorem 4.1.

4.1.1. Oscillations and image processing. As noted earlier, there exists no linear construction of solutions of (4.1) for general $f \in L^{2}$. Yet, for the 'slightly smaller' Lorenz space, $L^{2,1}$, we have

$$
\nabla \Delta^{-1} f \in L^{\infty}, \quad f \in L_{\#}^{2,1}\left(\mathbb{T}^{2}\right) .
$$

(we note in passing that $L^{2,1}$ is a limiting case for the linearity of $f \mapsto U$ to survive the $L^{2, \infty}$-based nonlinearity result argued in the proof of [BB03, proposition 2]). Thus, the nonlinear aspect of constructing hierarchical solutions for (4.1) becomes essential for highly oscillatory functions such that $f \in L^{2} \backslash L^{2,1}$ (and in particular, $f \notin B V\left(\mathbb{T}^{2}\right)$ ). Such $f$ 's are encountered in image processing in the form of noise, texture, and blurry images, [Me02, BCM05]. Hierarchical decompositions in this context of images were introduced by us in [TNV04] and were found to be effective tools in image de-noising, image de-blurring and image registration, [BCM05, LPSX06, PL07, TNV08, HRC10, AXRNW13, TH13], including graph-based signals [HLTE10, HLE13]. Here, we are given a noisy and possibly blurry observed image, $f=\mathscr{L} U+r \in L^{2}\left(\mathbb{R}^{2}\right)$, and the purpose is to recover a faithful description of the underlying 'clean' image, $U \sim$ “ $\mathscr{L}^{-1}$ " $f$, by de-noising $r$ and de-blurring $\mathscr{L}$. The inverse " $\mathscr{L}^{-1}$ " $f$ should be properly interpreted, say, in the smaller space $B V\left(\mathbb{R}^{2}\right) \subset L^{2}\left(\mathbb{R}^{2}\right)$ which is known to be well-adapted to represent edges. The resulting inverse problem can solved by corresponding variational problem of [ROF92, CL95, CL97]

$$
[\mathbf{u}, r]=\underset{\mathscr{L} \mathbf{u}+r=f}{\arg \min }\left\{\|\mathbf{u}\|_{B V}+\lambda\|r\|_{L^{2}\left(\mathbb{R}^{2}\right)}^{2}\right\},
$$

which is a special case of Tikhonov-regularization, [TA77, Mo84, Mo93]. The $\left(B V, L^{2}\right)$-hierarchical decomposition corresponding to (4.11) reads, [TNV04, TNV08],

$$
f \cong \mathscr{L} U_{m}, \quad U_{m}=\sum_{j=1}^{m} \mathbf{u}_{j}, \quad\left[\mathbf{u}_{j+1}, r_{j+1}\right]=\underset{\mathscr{L} \mathbf{u}+r=r_{j}}{\operatorname{argmin}}\left\{\|\mathbf{u}\|_{B V}+\lambda_{1} 2^{j}\|r\|_{L^{2}}^{2}\right\} .
$$

The oscillatory nature of noise and texture in images was addressed by Y. Meyer who advocated, [Me02], to replace $L^{2}$ with the larger space of "images" $G:=\left\{f \mid \operatorname{div} \mathbf{u}=f, \mathbf{u} \in L^{\infty}\right\}$. The equation $\operatorname{div} \mathbf{u}=f$ arises here with one-signed measure $f$ 's, and its $L^{\infty}$ solutions were characterized in [Me02, $\S 1.14],[\mathrm{PT} 08]$ : the space $G_{+}$coincides with Morrey space $M_{+}^{2}(\Omega)$ :

$$
M^{2}(\Omega)=\left\{\mu \in \mathscr{M} \mid \int_{B_{r}} d \mu \lesssim r, \quad \forall B_{r} \subset \Omega\right\} .
$$

For one-signed measure, $M_{+}^{2}(\Omega)$ coincides with Besov space $\dot{B}_{\infty}^{-1, \infty}$. The corresponding Meyer's energy functional then reads, $[\mathbf{u}, r]=\underset{\mathscr{L} \mathbf{u}+r=f}{\arg \min }\left\{\|\mathbf{u}\|_{B V(\Omega)}+\lambda\|r\|_{\dot{B}_{\infty}^{-1, \infty}(\Omega)}\right\}$; numerical simulations with this model are found in [VO04]. 
4.1.2. $L^{1}\left(\mathbb{T}^{2}\right)$-bounds and $\dot{H}^{-1}\left(\mathbb{T}^{2}\right)$-compactness. Here is a simple application of theorem 4.1 . Let $f \in \dot{H}^{-1}\left(\mathbb{T}^{2}\right)$ be given. For arbitrary $g \in \dot{H}^{1}\left(\mathbb{T}^{2}\right)$ we have $\xi_{j} \widehat{g}(\xi) \in L_{\#}^{2}\left(\mathbb{T}^{2}\right)$, and by theorem 4.1 , there exist bounded $U_{i j} \in L^{\infty}\left(\mathbb{T}^{2}\right)$, such that

$$
\left\{\begin{array}{l}
\xi_{1} \widehat{g}(\xi)=\xi_{1} \widehat{U}_{11}(\xi)+\xi_{2} \widehat{U}_{12}(\xi), \\
\xi_{2} \widehat{g}(\xi)=\xi_{1} \widehat{U}_{21}(\xi)+\xi_{2} \widehat{U}_{22}(\xi),
\end{array} \quad\left\|U_{i j}\right\|_{L^{\infty}} \lesssim\|g\|_{\dot{H}^{1}\left(\mathbb{T}^{2}\right)} .\right.
$$

Thus, expressed in terms of the Riesz transforms, $\widehat{R_{j} \psi}(\xi):=\widehat{\psi}(\xi) \xi_{j} /|\xi|$, we have

$$
g=\frac{1}{2}\left(U_{11}+U_{22}\right)+\frac{1}{2}\left(R_{1}^{2}-R_{2}^{2}\right)\left(U_{11}-U_{22}\right)+R_{1} R_{2}\left(U_{12}+U_{21}\right) ;
$$

Since $R_{1}^{2}-R_{2}^{2}$ and $R_{1} R_{2}$ agree up to rotation, we conclude that: every $g \in \dot{H}^{1}\left(\mathbb{T}^{2}\right)$ can be written as the sum

$$
g=U_{1}+R_{1} R_{2} U_{2}, \quad\left\|U_{1}\right\|_{L^{\infty}}+\left\|U_{2}\right\|_{L^{\infty}} \lesssim\|g\|_{\dot{H}^{1}\left(\mathbb{T}^{2}\right)} \quad \text { for all } g \in \dot{H}^{1}\left(\mathbb{T}^{2}\right) .
$$

Here, $U_{1}, U_{2}$ are given by linear combination of the $U_{i j}$ 's in their Cartesian and their rotated coordinates. The last representation shows that although an $L^{1}\left(\mathbb{T}^{2}\right)$-bound of $f$ does not imply $f \in \dot{H}^{-1}\left(\mathbb{T}^{2}\right)$, then $f$ does belong to $\dot{H}^{-1}$ if $f$ and its repeated Riesz transform, $R_{1} R_{2} f$, are $L^{1}$-bounded.

\section{Corollary 4.3. The following bound holds}

$$
\|f\|_{\dot{H}^{-1}\left(\mathbb{T}^{2}\right)} \lesssim\|f\|_{L^{1}\left(\mathbb{T}^{2}\right)}+\left\|R_{1} R_{2} f\right\|_{L^{1}\left(\mathbb{T}^{2}\right)} .
$$

As an example, consider a family of divergence-free 2-vector fields, $\mathbf{u}^{\varepsilon}(t, \cdot) \in L^{2}\left(\mathbb{T}^{2}, \mathbb{R}^{2}\right)$, which are approximate solutions of two-dimensional incompressible Euler's equations. One is interested in their convergence to a proper weak solution, with no concentration effects, [DM87]. It was shown in [LNT00] that $\left\{\mathbf{u}^{\varepsilon}\right\}$ converges to such a weak solution if the vorticity, $\omega^{\varepsilon}(t \cdot):=\partial_{1} u_{2}^{\varepsilon}(t, \cdot)-\partial_{2} u_{1}^{\varepsilon}(t, \cdot)$, is compactly embedded in $H^{-1}\left(\mathbb{T}^{2}\right)$. By corollary $4.3, H^{-1}$-compactness holds if $\left\{R_{1} R_{2} \omega^{\varepsilon}(t, \cdot)\right\} \hookrightarrow$ $L^{1}\left(\mathbb{T}^{2}\right)$; consult [Ve92].

4.2. Hierarchical solutions for $\mathscr{L} U=f \in L_{\#}^{p}(\Omega)$ : approximate solutions. We turn our attention to the construction of hierarchical solutions for equations of the general form

$$
\mathscr{L} U=f, \quad f \in L_{\#}^{p}(\Omega), \quad 1<p<\infty .
$$

A solution $U$ is sought in a Banach space $\mathbb{B}:=\left\{U:\|U\|_{\mathbb{B}}<\infty\right\}$. The general framework, involving two linear operators, $\mathscr{L}$ and $\mathscr{P}$, is outlined below.

The linear operator $\mathscr{L}$ is densely defined on $\mathbb{B}$ with a closed range in $L_{\#}^{p}:=L^{p} \cap \operatorname{Ker}(\mathscr{P})$ with appropriate $\mathscr{P}: L^{p} \mapsto L^{p}$. We let $\mathscr{L}^{*}: L^{p^{\prime}} \mapsto \mathbb{B}^{*}$ denote the formal dual of $\mathscr{L}$, acting on $L^{p^{\prime}}$ with the natural pairing (effectively, $\mathscr{L}^{*}$ is acting on $L_{\#}^{p^{\prime}}:=L^{p^{\prime}} \cap \operatorname{Ker}(\mathscr{P})$, since $R\left(\mathscr{P}^{*}\right)$ is in the null of $\mathscr{L}^{*}$ )

$$
\left\langle\mathscr{L}^{*} g, \mathbf{u}\right\rangle=(g, \mathscr{L} \mathbf{u}), \quad g \in L^{p^{\prime}}, \mathbf{u} \in \mathbb{B},
$$

and let $\|\cdot\|_{\mathbb{B}^{*}}$ denote the dual norm

$$
\left\|\mathscr{L}^{*} g\right\|_{\mathbb{B}^{*}}:=\sup _{\mathbf{u} \neq 0} \frac{\left\langle\mathscr{L}^{*} g, \mathbf{u}\right\rangle}{\|\mathbf{u}\|_{\mathbb{B}}}, \quad g \in L^{p^{\prime}} .
$$

We begin by constructing an approximate solution of (4.14), $U_{\lambda}: \mathscr{L} U_{\lambda} \approx f$, such that the residual $r_{\lambda}:=f-\mathscr{L} U_{\lambda}$ is driven to be small by a proper choice of a scaling parameter $\lambda$ at our disposal. The approximate solution is obtained in terms of minimizers of the variational problem,

$$
\vee(f, \lambda):=\inf _{\mathscr{L} \mathbf{u}+r=f}\left\{\|\mathbf{u}\|_{\mathbb{B}}+\lambda\|r\|_{L^{p}}^{p}: \mathbf{u} \in \mathbb{B}, r \in L_{\#}^{p}\right\} .
$$


In theorem 5.1 below we show if $\lambda$ is chosen sufficiently large,

$$
\lambda>\frac{1}{p\left\|\mathscr{L}^{*} \varphi(f)\right\|_{\mathbb{B}^{*}}}, \quad \varphi(f):=\operatorname{sgn}(f)|f|^{p-1},
$$

then the functional $\vee(f, \lambda)$ in (4.15) admits a minimizer, $\mathbf{u}=\mathbf{u}_{\lambda}$, such that the size of the residual, $r_{\lambda}:=f-\mathscr{L} \mathbf{u}_{\lambda}$, is dictated by the dual statement

$$
\left\|\mathscr{L}^{*} \varphi\left(r_{\lambda}\right)\right\|_{\mathbb{B}^{*}}=\frac{1}{p \lambda} .
$$

Fix the scale $\lambda=\lambda_{1}>1 / p\left\|\mathscr{L}^{*} \varphi(f)\right\|_{\mathbb{B}^{*}}$. We construct an approximate solution, $\mathscr{L}_{1} \approx f, U_{1}:=$ $\mathbf{u}_{1}$, where $\mathbf{u}_{1}$ is a minimizer of $\vee\left(f, \lambda_{1}\right)$,

$$
f=\mathscr{L} \mathbf{u}_{1}+r_{1}, \quad\left[\mathbf{u}_{1}, r_{1}\right]=\underset{\mathscr{L} \mathbf{u}+r=f}{\arg \min } \vee\left(f, \lambda_{1}\right)
$$

Borrowing the terminology from image processing we note that the corresponding residual $r_{1}$ contains 'small' features which were left out of $\mathbf{u}_{1}$. Of course, whatever is interpreted as 'small' features at a given $\lambda_{1}$-scale, may contain significant features when viewed under a refined scale, say $\lambda_{2}>\lambda_{1}$. To this end we assume that the residual $r_{1} \in L_{\#}^{p}$ so that we can repeat the $\vee$-decomposition of $r_{1}$, this time at the refined scale $\lambda_{2}$ :

$$
r_{1}=\mathscr{L} \mathbf{u}_{2}+r_{2}, \quad\left[\mathbf{u}_{2}, r_{2}\right]=\underset{\mathscr{L} \mathbf{u}+r=r_{1}}{\arg \min } \vee\left(r_{1}, \lambda_{2}\right)
$$

Combining the last two steps we arrive at a better two-scale representation of $U$ given by $U_{2}:=\mathbf{u}_{1}+$ $\mathbf{u}_{2}$, as an improved approximate solution of $\mathscr{L} U_{2} \approx f$. Features below scale $\lambda_{2}$ remain unresolved in $U_{2}$, but the process can be continued by successive application of the refinement step,

$$
r_{j}=\mathscr{L} \mathbf{u}_{j+1}+r_{j+1}, \quad\left[\mathbf{u}_{j+1}, r_{j+1}\right]:=\underset{\mathscr{L} \mathbf{u}+r_{=} r_{j}}{\arg \min } \vee\left(r_{j}, \lambda_{j+1}\right), \quad j=1,2, \ldots
$$

To enable this process we require the residuals $r_{j}$ to remain in $L_{\#}^{p}$. In view of the dual bound (4.17), we therefore make the following

Assumption (A closure bound). There exists a constant $\eta=\eta(p, d)<\infty$ such that the following a priori estimate holds

$$
\|g\|_{L^{p}}^{p} \leq \eta\left\|\mathscr{L}^{*} \varphi(g)\right\|_{\mathbb{B}^{*}}^{p^{\prime}}, \quad \varphi(g)=\operatorname{sgn}(g)|g|^{p-1} .
$$

We postpone the discussion of this bound to theorem 4.4 below and we continue with the generic hierarchical step where $\left[\mathbf{u}_{j+1}, r_{j+1}\right]$ is constructed as a minimizing pair of $\vee\left(r_{j}, \lambda_{j+1}\right)$ : since this minimizer is characterized by satisfying $\left\|\mathscr{L}^{*} \varphi\left(r_{j+1}\right)\right\|_{\mathbb{B}^{*}}=1 / p \lambda_{j+1}$, then the closure bound (4.19) implies that $r_{j+1} \in L^{p}$; moreover, since $r_{j}$ and $R(\mathscr{L})$ are in $\operatorname{Ker}(\mathscr{P})$ then,

$$
r_{j+1}=r_{j}-\mathscr{L} \mathbf{u}_{j+1} \in \operatorname{Ker}(\mathscr{P}),
$$

and we conclude that $r_{j+1} \in L_{\#}^{p}$. In this manner, the iteration step $\left[\mathbf{u}_{j}, r_{j}\right] \mapsto\left[\mathbf{u}_{j+1}, r_{j+1}\right]$, is welldefined on $\mathbb{B} \times L_{\#}^{p}$. After $k$ such steps we have,

$$
\begin{aligned}
f & =\mathscr{L} \mathbf{u}_{1}+r_{1}= \\
& =\mathscr{L} \mathbf{u}_{1}+\mathscr{L} \mathbf{u}_{2}+r_{2}= \\
& =\cdots \ldots= \\
& =\mathscr{L} \mathbf{u}_{1}+\mathscr{L} \mathbf{u}_{2}+\cdots+\mathscr{L} \mathbf{u}_{k}+r_{k} .
\end{aligned}
$$


We end up with a multiscale hierarchical representation of an approximate solution of (4.14) $U_{k}:=$ $\sum_{j=1}^{k} \mathbf{u}_{j} \in \mathbb{B}$ such that $\mathscr{L} U_{k} \cong f$. Here, the approximate equality $\cong$ is interpreted as the convergence of the residuals,

$$
\left\|\mathscr{L}^{*} \varphi\left(r_{k}\right)\right\|_{\mathbb{B}^{*}}=\frac{1}{\lambda_{k}} \rightarrow 0, \quad r_{k}:=f-\mathscr{L} U_{k}
$$

dictated by the sequence of scales, $\lambda_{1}<\lambda_{2}<\ldots<\lambda_{k}$, which is at our disposal. We summarize with the following theorem.

Theorem 4.4 (Approximate solutions). Consider $\mathscr{L}: \mathbb{B} \mapsto L_{\#}^{p}(\Omega)$ and assume its dual is injective so that (1.3) holds,

$$
\left\|g-\mathscr{P}^{*} g\right\|_{L^{p^{\prime}}} \leq \beta\left\|\mathscr{L}^{*} g\right\|_{\mathbb{B}^{*}}, \quad \forall g \in L^{p^{\prime}}(\Omega),
$$

for some $\mathscr{P}: L^{p} \mapsto L^{p}$ whose range is "compatible" with the range of $\mathscr{L}$. Then, the equation $\mathscr{L} U=$ $f \in L_{\#}^{p}(\Omega)$ admits an approximate solution, $U_{k} \in \mathbb{B}$, such that $\mathscr{L} U_{k} \cong f$ in the sense that the residuals $r_{k}:=f-\mathscr{L} U_{k}$ satisfy

$$
\left\|\mathscr{L}^{*} \varphi\left(r_{k}\right)\right\|_{\mathbb{B}^{*}}=\frac{1}{p \lambda_{k}}, \quad r_{k}:=f-\mathscr{L} U_{k} .
$$

The approximate solution admits the hierarchical expansion, $U_{k}=\sum_{j=1}^{k} \mathbf{u}_{j}$, where the $\mathbf{u}_{j}$ 's are constructed as minimizers,

$$
\left[\mathbf{u}_{j+1}, r_{j+1}\right]=\underset{\mathscr{L} \mathbf{u}+r=r_{j}}{\operatorname{argmin}}\left\{\|\mathbf{u}\|_{\mathbb{B}}+\lambda_{j+1}\|r\|_{L^{p}}^{p}\right\}, \quad r_{0}=f .
$$

Proof. We verify that the a priori duality estimate assumed in (1.3) implies the closure bound sought in (4.19). Fix $g \in L_{\#}^{p}(\Omega)$. Then $\varphi(g):=\operatorname{sgn}(g)|g|^{p-1} \in L^{p^{\prime}}(\Omega)$ and since $g \in \operatorname{Ker}(\mathscr{P})$ we find

$$
\int_{\Omega}|g|^{p} d x=\int_{\Omega} g \varphi(g) d x=\int_{\Omega} g\left(\varphi(g)-\mathscr{P}^{*} \varphi(g)\right) d x \leq\|g\|_{L^{p}}\left\|\varphi(g)-\mathscr{P}^{*} \varphi(g)\right\|_{L^{p^{\prime}}} .
$$

The a priori dual estimate assumed in (1.3) then yields

$$
\|g\|_{L^{p}}^{p} \leq\|g\|_{L^{p}}\left\|\varphi(g)-\mathscr{P}^{*} \varphi(g)\right\|_{L^{p^{\prime}}} \leq \beta\|g\|_{L^{p}}\left\|\mathscr{L}^{*} \varphi(g)\right\|_{\mathbb{B}^{*}}, \quad \forall g \in L_{\#}^{p}(\Omega),
$$

and the closure bound (4.19) follows with $\eta:=\beta^{p^{\prime}}$,

$$
\|g\|_{L^{p}}^{p-1} \leq \beta\left\|\mathscr{L}^{*} \varphi(g)\right\|_{\mathbb{B}^{*}}
$$

This allows us to proceed with the hierarchical iterations (4.18),

$$
\left[\mathbf{u}_{j}, r_{j}\right] \in \mathbb{B} \times L_{\#}^{p} \mapsto\left[\mathbf{u}_{j+1}, r_{j+1}\right]:=\underset{\mathscr{L} \mathbf{u}+r=r_{j}}{\arg \min } \vee\left(r_{j}, \lambda_{j+1}\right) \in \mathbb{B} \times L_{\#}^{p}, \quad j=1,2, \ldots
$$

starting with $\left[\mathbf{u}_{0}, r_{0}\right]=[0, f]$. A telescoping summation of (4.18) yields an approximate solution $U_{k}=\sum_{j=1}^{k} \mathbf{u}_{j}$ such that its residual $r_{k}=f-\mathscr{L} U_{k}$ satisfies (4.20).

Remark 4.2 (on the closure bound). As an example for the closure bound (4.21) for $\mathscr{L}$ 's with an injective dual, consider the critical case of $\mathscr{L}=\operatorname{div}: L^{\infty} \mapsto L^{d}\left(\mathbb{T}^{d}\right)$ and let $\mathscr{P}$ denote the zero averaging projection $\mathscr{P} g=g-\bar{g}$. The corresponding dual estimate (1.3) reads

$$
\|g-\bar{g}\|_{L^{d^{\prime}}} \lesssim\left\|\mathscr{L}^{*} g\right\|_{\dot{W}^{1,1}}
$$

This is the isoperimetric Gagliardo-Nirenberg inequality and it implies, along the lines of theorem 4.4 , the following closure bound corresponding to (4.19)

$$
\|g\|_{L^{d}\left(\mathbb{T}^{d}\right)}^{d-1} \lesssim\left\|\operatorname{sgn}(g)|g|^{d-1}\right\|_{\dot{W}^{1,1}\left(\mathbb{T}^{d}\right)}, \quad \forall g \in L_{\#}^{d}\left(\mathbb{T}^{d}\right) .
$$


Equivalently, we can rewrite this as $\|\varphi(g)\|_{L^{d^{\prime}}} \lesssim\|\varphi(g)\|_{\dot{W}^{1,1}\left(\mathbb{T}^{d}\right)}$. The observant reader will notice that the latter is a slight variant of Gagliardo-Nirenberg inequality since for $d>2, \varphi(g)$ need not have zero average; only $g$ does.

4.3. From approximate to exact solutions. We turn to show that the approximate solutions, $U_{k}=$ $\sum_{j=1}^{k} \mathbf{u}_{j}$, converge to a limit $U=\sum_{j=1}^{\infty} \mathbf{u}_{j}$, which is an exact solution sought for (4.14), uniformly bounded in $\mathbb{B}$.

We start by comparing the minimizer $\left[\mathbf{u}_{j+1}, r_{j+1}\right]$ of $\vee\left(r_{j}, \lambda_{j+1}\right)$ in (4.18) with the trivial pair $\left[\mathbf{u} \equiv 0, r_{j}\right]$, which yields the key refinement estimate

$$
\left\|r_{j}\right\|_{L^{p}}^{p} \geq \frac{1}{\lambda_{j+1}}\left\|\mathbf{u}_{j+1}\right\|_{\mathbb{B}}+\left\|r_{j+1}\right\|_{L^{p}}^{p}, \quad j=0,1, \ldots
$$

In particular, the closure bound (4.19) followed by (4.20) imply

$$
\left\|\mathbf{u}_{j+1}\right\|_{\mathbb{B}} \leq \lambda_{j+1}\left\|r_{j}\right\|_{L^{p}}^{p} \begin{cases}=\lambda_{1}\|f\|_{L^{p}}^{p}, & j=0, \\ \leq \lambda_{j+1} \eta\left\|\mathscr{L}^{*} \varphi\left(r_{j}\right)\right\|_{\mathbb{B}^{*}}^{1 / p^{\prime}} \leq \frac{\lambda_{j+1} \eta}{\left(p \lambda_{j}\right) p^{\prime}}, & j=1,2, \ldots,\end{cases}
$$

where $\left\{\lambda_{j}\right\}$ is an increasing sequence of scales at our disposal. Setting $\lambda_{j}=\lambda_{1} 2^{j-1}$, we conclude that the approximate solutions, $U_{k}=\sum_{1}^{k} \mathbf{u}_{j}$ form a Cauchy sequence,

$$
\left\|U_{k}-U_{\ell}\right\|_{\mathbb{B}} \lesssim \sum_{j=\ell+1}^{k} 2^{j\left(1-p^{\prime}\right)} \ll 1, \quad k>\ell \gg 1,
$$

which has a limit, $U=\sum_{j=1}^{\infty} \mathbf{u}_{j}$, such that $\left\|\mathscr{L} U_{j}-f\right\|_{L^{p}}^{p} \rightarrow 0$. Since $\mathscr{L}$ has a closed range, $\mathscr{L} U=f$. It remains to show that the limit $U$ has a finite $\mathbb{B}$-norm, which brings us to

Proof of theorem 1.1. Using (4.23) with $\eta=\beta^{p^{\prime}}$ yields

$$
\|U\|_{\mathbb{B}} \leq\left\|\mathbf{u}_{1}\right\|_{\mathbb{B}}+\sum_{j=1}^{\infty}\left\|\mathbf{u}_{j+1}\right\|_{\mathbb{B}} \leq \lambda_{1}\|f\|_{L^{p}}^{p}+\sum_{j=1}^{\infty} \frac{\lambda_{1} 2^{j} \eta}{\left(p \lambda_{1} 2^{j-1}\right)^{p^{\prime}}} \leq \lambda_{1}\|f\|_{L^{p}}^{p}+\left(\frac{2 \beta}{p}\right)^{p^{\prime}} \frac{1}{\lambda_{1}^{p^{\prime}-1}\left(2^{p^{\prime}-1}-1\right)} .
$$

Set $\lambda_{1}:=\frac{2 \beta}{p}\|f\|_{L^{p}}^{1-p}$. Such a choice of $\lambda_{1}$ satisfies the admissibility requirement (4.16): indeed, according to (4.19), $\|g\|_{L^{p}}^{p-1} \leq \beta\left\|\mathscr{L}^{*} \varphi(g)\right\|_{\mathbb{B}^{*}}$, hence

$$
\lambda_{1}=\frac{2 \beta}{p}\|f\|_{L^{p}}^{1-p}>\frac{1}{p\left\|\mathscr{L}^{*} \varphi(f)\right\|_{\mathbb{B}^{*}}},
$$

and the uniform bound (1.4) follows,

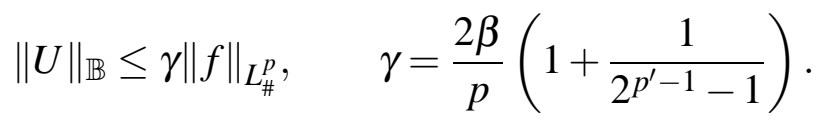

Remark 4.3. We summarize the two main aspects in the hierarchical construction.

(i) The existence minimizers $\left\{\mathbf{u}_{j}\right\}$ of $\vee\left(r_{j-1}, \lambda_{j}\right)$, which follows from basic principles in uniformly convex Banach spaces. We use here the mere existence of such minimizers, $\left\{\mathbf{u}_{j}\right\}$, instead of standard duality-based existence arguments in the closed range theorem, e.g., [Yos80, VII.5],[Woj91, I.A.1314],[Bre10, theorem 2.20]. We note in passing that existence of minimizers and duality principles in uniformly convex Banach spaces can be deduced from each other, [Jam47]. 
(ii) The exponential decay of these minimizers and hence the uniform bound of their sum, $\|U\|_{\mathbb{B}} \leq$ $\sum\left\|\mathbf{u}_{j}\right\|_{\mathbb{B}} \lesssim\|f\|_{L_{\#}^{p}}$, follow from the key a priori dual estimate (1.3) used in the refinement step (4.23).

Remark 4.4 (Extension to Orlicz spaces). The hierarchical construction extends to equations valued in more general Orlicz spaces,

$$
\mathscr{L} U=f \in L_{\#}^{\Phi}:=L^{\Phi} \cap \operatorname{Ker}\left(\mathscr{P}^{*}\right), \quad L^{\Phi}=\left\{f:[f]_{\Phi}:=\int_{\Omega} \Phi(|f|) d x<\infty\right\},
$$

for a proper $N$-function $\Phi$, satisfying the $\Delta_{2}$ condition, [AF03, §8],[BS88, §4.8]. Assume that the following a priori closure bound holds: there exists an increasing function $\eta: \mathbb{R}_{+} \mapsto \mathbb{R}_{+}$such that

$$
[g]_{\Phi} \leq \eta\left(\left\|\mathscr{L}^{*} \varphi(g)\right\|_{\mathbb{B}}\right), \quad \int_{s=0}^{1} \frac{\eta(s)}{s^{2}} d s<\infty .
$$

Then, the problem (4.25) admits the bounded hierarchical solution, $U=\sum \mathbf{u}_{j}$, such that $\|U\|_{\mathbb{B}} \lesssim[f]_{\Phi}$. The closure bound enters through the initial scale $\lambda_{1} \gtrsim 1 / \eta^{-1}\left([f]_{\Phi}\right)$. The $L^{p}$ setup corresponds to $\Phi(t)=t^{p}$ and $\eta(s) \sim s^{p^{\prime}}$.

Remark 4.5 (Sharp bounds). The bound (4.24) with $p=2$ shows that if $\mathscr{L}^{*}$ is injective so that (1.3) holds with constant $\beta$, then $\mathscr{L} U=f \in L^{2}$ admits a solution $\|U\|_{\mathbb{B}} \leq \gamma\|f\|_{L^{2}}$, with twice the bound $\gamma=2 \beta$ (in agreement with the $L^{2}$-case in theorem (4.1)). Using a rapidly growing scales, $\lambda_{j+1}=\lambda_{1} \zeta^{j}$ with $\zeta \gg 1$ yields a tighter bound $\gamma$. A sharp form of the $\mathbb{B}$-bound (4.24) for general $1<p<\infty$,

$$
\|U\|_{\mathbb{B}} \leq \gamma\|f\|_{L_{\#}^{p}} \text { for any } \quad \gamma>\beta
$$

can be argued by Hahn-Banach theorem. To this end, we reproduce here a slight generalization of [BB03, proposition 1]. Normalize $\|f\|_{L^{p}}=1$ and consider the two non-empty convex sets: the ball

$$
B_{\gamma_{\varepsilon}}:=\left\{\mathbf{u} \in \mathbb{B}:\|\mathbf{u}\|_{\mathbb{B}}<\gamma_{\varepsilon}\right\}, \quad \gamma_{\varepsilon}:=(1+\varepsilon) \beta,
$$

and $C:=\{U \in \mathbb{B}: \mathscr{L} U=f\}$. The claim is that $B_{\gamma_{\varepsilon}} \cap C \neq \emptyset$ and the desired estimate (4.26) $\gamma_{\varepsilon}$ then follows with arbitrarily small $\varepsilon$. If not, $B_{\gamma_{\varepsilon}} \cap C=\emptyset$, and by Hahn-Banach there exists a non-trivial $g^{*} \in L^{p^{\prime}}$ such that for some $\alpha \in \mathbb{R}_{+}$

$$
\left\langle g^{*}, \mathbf{u}\right\rangle \leq \alpha, \quad \forall \mathbf{u} \in B_{\gamma_{\varepsilon}}
$$

and

$$
\left\langle g^{*}, U\right\rangle \geq \alpha, \quad \forall U \in C .
$$

If $V \in \operatorname{Ker}(\mathscr{L})$ then application of (4.27b) with $U \mapsto U \pm \delta V \in C$ yields $\pm \delta\left\langle g^{*}, V\right\rangle \geq 0$, or $\left\langle g^{*}, V\right\rangle=0$; that is, $g^{*} \in \operatorname{Ker}(\mathscr{L})^{\perp}=\mathrm{R}\left(\mathscr{L}^{*}\right)$ is of the form $g^{*}=\mathscr{L}^{*} g$ for some $g \in D\left(\mathscr{L}^{*}\right) \subset L^{p^{\prime}}$. Now, by (4.27a)

$$
\left\|g^{*}\right\|_{\mathbb{B}^{*}}=\sup _{\|\mathbf{u}\|_{\mathbb{B}}=\gamma_{\varepsilon / 2}} \frac{\left\langle g^{*}, \mathbf{u}\right\rangle}{\gamma_{\varepsilon / 2}} \leq \frac{\alpha}{\gamma_{\varepsilon / 2}},
$$

and the a priori estimate assumed in (1.3) implies

$$
\|g\|_{L_{\#}^{p^{\prime}}} \leq \beta\left\|\mathscr{L}^{*} g\right\|_{\mathbb{B}^{*}}=\beta\left\|g^{*}\right\|_{\mathbb{B}^{*}} \leq \frac{\alpha}{1+\varepsilon / 2} .
$$

But this leads to a contradiction: pick $U \in C$ (which we recall is not empty) then (4.27b) implies,

$$
\alpha \leq\left\langle g^{*}, U\right\rangle=\left\langle\mathscr{L}^{*} g, U\right\rangle=\langle g, f\rangle \leq\|g\|_{L^{p^{\prime}}}\|f\|_{L^{p}} \leq \frac{\alpha}{1+\varepsilon / 2} .
$$




\section{AN APPENDIX ON $\vee$-MINIMIZERS}

To study the hierarchical expansions (4.18), we characterize the minimizers of the $\vee$-functionals (4.15)

$$
[\mathbf{u}, r]:=\underset{\mathscr{L} \mathbf{u}+r=f}{\arg \min } \vee(f, \lambda), \quad \vee(f, \lambda):=\inf _{\mathscr{L} \mathbf{u}+r=f}\left\{\|\mathbf{u}\|_{\mathbb{B}}+\lambda\|r\|_{L^{p}}^{p}: \mathbf{u} \in \mathbb{B}\right\} .
$$

Here $\mathscr{L}: \mathbb{B} \mapsto L_{\#}^{p}(\Omega)$ is densely defined into a subspace of $L^{p}(\Omega)$ over a Lipschitz domain $\Omega \subset$ $\mathbb{R}^{d}$. The characterization summarized below extends related results which can be found in $[\mathrm{Me} 02$, Theorem 4], [ACM04, Chapter1], [TNV08, Theorem 2.3].

Recall that $\|\cdot\|_{\mathbb{B}^{*}}$ denotes the dual norm, $\left\|\mathscr{L}^{*} g\right\|_{\mathbb{B}^{*}}=\sup _{\mathbf{u}}\left\langle\mathscr{L}^{*} g, \mathbf{u}\right\rangle /\|\mathbf{u}\|_{\mathbb{B}}$, so that the usual duality bound holds

$$
\left\langle\mathscr{L}^{*} g, \mathbf{u}\right\rangle \leq\|\mathbf{u}\|_{\mathbb{B}}\left\|\mathscr{L}^{*} g\right\|_{\mathbb{B}^{*}}, \quad g \in L^{p^{\prime}}, \mathbf{u} \in \mathbb{B} .
$$

We say that $\mathbf{u}$ and $\mathscr{L}^{*} g$ is an extremal pair if equality holds above. The theorem below characterizes $[\mathbf{u}, r]$ as a minimizer of the $\vee$-functional if and only if $\mathbf{u}$ and $\mathscr{L}^{*} \varphi(r)$ form an extremal pair.

Theorem 5.1. Let $\mathscr{L}: \mathbb{B} \rightarrow L_{\#}^{p}(\Omega)$ be a linear operator with dual $\mathscr{L}^{*}$ and let $\vee(f, \lambda)$ denote the associated functional (4.15).

(i) The variational problem (5.1) admits a minimizer $\mathbf{u}$. Moreover, if $\|\cdot\|_{\mathbb{B}}$ is strictly convex, then the minimizer $\mathbf{u}$ is unique.

(ii) $\mathbf{u} \in \mathbb{B}$ is a minimizer of (5.1) if and only if the residual $r:=f-\mathscr{L} \mathbf{u}$ satisfies

$$
\left\langle\mathscr{L}^{*} \varphi(r), \mathbf{u}\right\rangle=\|\mathbf{u}\|_{\mathbb{B}} \cdot\left\|\mathscr{L}^{*} \varphi(r)\right\|_{\mathbb{B}^{*}}=\frac{\|\mathbf{u}\|_{\mathbb{B}}}{p \lambda}, \quad \varphi(r):=\operatorname{sgn}(r)|r|^{p-1} \in L^{p^{\prime}} .
$$

Proof. (i) The existence of a minimizer for the $\vee$-functional follows from standard arguments which we omit, consult [AV94, Me02]. We address the issue of uniqueness. Assume $\mathbf{u}_{1}$ and $\mathbf{u}_{2}$ are minimizers with the corresponding residuals $r_{1}=f-\mathscr{L} \mathbf{u}_{1}$ an $r_{2}=f-\mathscr{L} \mathbf{u}_{2}$

$$
\left\|\mathbf{u}_{i}\right\|_{\mathbb{B}}+\lambda\left\|r_{i}\right\|_{L^{p}}^{p}=v_{\min }, \quad i=1,2
$$

We then end up with the one-parameter family of minimizers, $\mathbf{u}_{\theta}:=\mathbf{u}_{1}+\theta\left(\mathbf{u}_{2}-\mathbf{u}_{1}\right), \theta \in[0,1]$,

$$
v_{\text {min }} \leq\left\|\mathbf{u}_{\theta}\right\|_{\mathbb{B}}+\lambda\left\|r_{\theta}\right\|_{L^{p}}^{p} \leq \theta\left\|\mathbf{u}_{2}\right\|_{\mathbb{B}}+(1-\theta)\left\|\mathbf{u}_{1}\right\|_{\mathbb{B}}+\theta \lambda\left\|r_{2}\right\|_{L^{p}}^{p}+(1-\theta) \lambda\left\|r_{1}\right\|_{L^{p}}^{p}=v_{\text {min }} .
$$

Consequently, $\left\|r_{\theta}\right\|_{L^{p}}^{p}=\theta\left\|r_{2}\right\|_{L^{p}}^{p}+(1-\theta)\left\|r_{1}\right\|_{L^{p}}^{p}$ and hence $r_{1}=r_{2}$. In particular, $\left\|r_{1}\right\|_{L^{p}}^{p}=\left\|r_{2}\right\|_{L^{p}}^{p}$ implies that the two minimizers satisfy $\left\|\mathbf{u}_{1}\right\|_{\mathbb{B}}=\left\|\mathbf{u}_{2}\right\|_{\mathbb{B}}$ and we conclude that the ball $\|\mathbf{u}\|_{\mathbb{B}}=\left\|\mathbf{u}_{1}\right\|_{\mathbb{B}} \neq$ 0 contains the segment $\left\{\mathbf{u}_{\theta}, \theta \in[0,1]\right\}$, which by strict convexity, must be the trivial segment, i.e., $\mathbf{u}_{2}=\mathbf{u}_{1}$.

We note in passing that strict convexity is in fact necessary for uniqueness, e.g., the counterexample of lack of uniqueness over the $\ell^{\infty}$-unit ball, [Me02, pp. 40].

(ii) If $\mathbf{u}$ is a minimizer of (5.1) then for any $\mathbf{v} \in \mathbb{B}$ we have

$$
\begin{aligned}
& \vee(\mathbf{u}, \lambda)=\|\mathbf{u}\|_{\mathbb{B}}+\lambda\|f-\mathscr{L} \mathbf{u}\|_{L^{p}}^{p} \\
& \quad \leq \vee(\mathbf{u}+\varepsilon \mathbf{v}, \lambda)=\|\mathbf{u}+\varepsilon \mathbf{v}\|_{\mathbb{B}}+\lambda\|f-\mathscr{L}(\mathbf{u}+\varepsilon \mathbf{v})\|_{L^{p}}^{p} \\
& \quad \leq\|\mathbf{u}\|_{\mathbb{B}}+|\varepsilon| \cdot\|\mathbf{v}\|_{\mathbb{B}}+\lambda\|f-\mathscr{L} \mathbf{u}\|_{L^{p}}^{p}-\lambda \varepsilon p\left(\operatorname{sgn}(f-\mathscr{L} \mathbf{u})|f-\mathscr{L} \mathbf{u}|^{p-1}, \mathscr{L} \mathbf{v}\right)+o(\varepsilon)
\end{aligned}
$$

It follows that for all $\mathbf{v} \in \mathbb{B}$,

$$
\left|\left\langle\mathscr{L}^{*} \varphi(r), \mathbf{v}\right\rangle\right| \leq \frac{1}{p \lambda}\|\mathbf{v}\|_{\mathbb{B}}+o(1), \quad \varphi(r)=\operatorname{sgn}(r)|r|^{p-1}, r:=f-\mathscr{L} \mathbf{u}
$$


and by letting $\varepsilon \rightarrow 0$

$$
\left\|\mathscr{L}^{*} \varphi(r)\right\|_{\mathbb{B}^{*}} \leq \frac{1}{p \lambda} .
$$

To verify the reverse inequality, we set $\mathbf{v}= \pm \mathbf{u}$ and $0<\varepsilon<1$ in (5.4), yielding

$$
\|\mathbf{u}\|_{\mathbb{B}}+\lambda\|f-\mathscr{L} \mathbf{u}\|_{L^{p}}^{p} \leq(1 \pm \varepsilon)\|\mathbf{u}\|_{\mathbb{B}}+\lambda\|f-\mathscr{L} \mathbf{u} \mp \varepsilon \mathscr{L} \mathbf{u}\|_{L^{p}}^{p},
$$

and hence $\pm \varepsilon\|\mathbf{u}\|_{\mathbb{B}} \mp p \lambda \varepsilon(\varphi(f-\mathscr{L} \mathbf{u}), \mathscr{L} \mathbf{u})+o(\varepsilon) \geq 0$. Dividing by $\varepsilon$ and letting $\varepsilon \downarrow 0_{+}$, we obtain $\|\mathbf{u}\|_{\mathbb{B}}=p \lambda\left\langle\mathscr{L}^{*} \varphi(r), \mathbf{u}\right\rangle$ and (5.3) follows:

$$
\frac{1}{p \lambda}\|\mathbf{u}\|_{\mathbb{B}}=\left\langle\mathscr{L}^{*} \varphi(r), \mathbf{u}\right\rangle \leq\left\|\mathscr{L}^{*} \varphi(r)\right\|_{\mathbb{B}^{*}}\|\mathbf{u}\|_{\mathbb{B}} \leq \frac{1}{p \lambda}\|\mathbf{u}\|_{\mathbb{B}} .
$$

Conversely, we show that if (5.3) holds then $\mathbf{u}$ is a minimizer. The convexity of $L^{p}$ yields

$$
\begin{aligned}
& \| f- \\
& \quad \geq\|r\|_{L^{p}(\Omega)}^{p}-p(\mathbf{u}+\mathbf{v})\left\|_{L^{p}}^{p}=\right\| r-\mathscr{L} \mathbf{v} \|_{L^{p}}^{p}= \\
& \left.\quad=\| f-|r|^{p-1}, \mathscr{L}(\mathbf{u}+\mathbf{v})\right)+p(\operatorname{u} \|_{L^{p}}^{p}-p \overbrace{\left\langle\mathscr{L}^{*} \varphi(r),(\mathbf{u}+\mathbf{v})\right\rangle}^{\# 1}+p \overbrace{\left\langle\mathscr{L}^{*} \varphi(r), \mathbf{u}\right\rangle}^{\# 2} .
\end{aligned}
$$

By the equalities assumed in (5.3), $\left\|\mathscr{L}^{*} \varphi(r)\right\|_{\mathbb{B}^{*}}=1 / p \lambda$, which implies $-p \lambda(\# 1) \geq-\|\mathbf{u}+\mathbf{v}\|_{\mathbb{B}}$; moreover, $p \lambda(\# 2)=\|\mathbf{u}\|_{\mathbb{B}}$. We conclude that for any $\mathbf{v} \in \mathbb{B}$,

$$
\begin{aligned}
\vee(\mathbf{u}+\mathbf{v}, \lambda)=\|\mathbf{u}+\mathbf{v}\|_{\mathbb{B}}+\lambda\|f-\mathscr{L}(\mathbf{u}+\mathbf{v})\|_{L^{p}}^{p} & \geq\|\mathbf{u}+\mathbf{v}\|_{\mathbb{B}}+\lambda\|f-\mathscr{L} \mathbf{u}\|_{L^{p}}^{p}-p \lambda(\# 1)+p \lambda(\# 2) \\
& \geq\|\mathbf{u}\|_{\mathbb{B}}+\lambda\|f-\mathscr{L} \mathbf{u}\|_{L^{p}}^{p}=\vee(\mathbf{u}, \lambda) .
\end{aligned}
$$

Thus, $\mathbf{u}$ is a minimizer of (5.1).

The next two assertions are a refinement of Theorem 5.1, depending on the size of $\left\|\mathscr{L}^{*} \varphi(f)\right\|_{\mathbb{B}^{*}}$.

Lemma 5.2 (The case $\left.\left\|\mathscr{L}^{*} \varphi(f)\right\|_{\mathbb{B}^{*}} \leq 1 / p \lambda\right)$. Let $\mathscr{L}: \mathbb{B} \rightarrow L_{\#}^{p}(\Omega)$ with adjoint $\mathscr{L}^{*}$ and let $\vee$ denote the associated functional (4.15). Then $p \lambda\left\|\mathscr{L}^{*} \varphi(f)\right\|_{\mathbb{B}^{*}} \leq 1$ if and only if $\mathbf{u} \equiv 0$ is a minimizer of (5.1).

Proof. Assume $\left\|\mathscr{L}^{*} \varphi(f)\right\|_{\mathbb{B}^{*}} \leq 1 / p \lambda$. Then by convexity of $L^{p}$

$$
\begin{aligned}
\|\mathbf{u}\|_{\mathbb{B}}+\lambda\|f-\mathscr{L} \mathbf{u}\|_{L^{p}}^{p} & \geq\|\mathbf{u}\|_{\mathbb{B}}+\lambda \int_{\Omega}|f|^{p} d x-p \lambda \int_{\Omega}(\varphi(f), \mathscr{L} \mathbf{u}) d x \\
& \geq\|\mathbf{u}\|_{\mathbb{B}}+\lambda \int_{\Omega}|f|^{p} d x-p \lambda\left\|\mathscr{L}^{*} \varphi(f)\right\|_{\mathbb{B}^{*}}\|\mathbf{u}\|_{\mathbb{B}} \geq \lambda\|f\|_{L^{p}}^{p},
\end{aligned}
$$

which tells us that $\mathbf{u} \equiv 0$ is a minimizer of (4.15). Conversely, if $\mathbf{u} \equiv 0$ is a minimizer of (5.1), then $\varepsilon\|\mathbf{u}\|_{\mathbb{B}}+\lambda\|f-\varepsilon \mathscr{L} \mathbf{u}\|_{L^{p}}^{p} \geq\|f\|_{L^{p}}^{p}$ for all $\mathbf{u} \in \mathbb{B}$. It follows that

$$
\varepsilon\|\mathbf{u}\|_{\mathbb{B}}-\varepsilon p \lambda \int_{\Omega}(\varphi(f), \mathscr{L} \mathbf{u}) d x+o(\varepsilon) \geq 0
$$

Letting $\varepsilon \downarrow 0$ we conclude $p \lambda\left\langle\mathscr{L}^{*} \varphi(f), \mathbf{u}\right\rangle \leq\|\mathbf{u}\|_{\mathbb{B}}$, hence $\left\|\mathscr{L}^{*} \varphi(f)\right\|_{\mathbb{B}^{*}} \leq 1 / p \lambda$.

Lemma 5.3 (The case $\left.\left\|\mathscr{L}^{*} \varphi(f)\right\|_{\mathbb{B}^{*}}>1 / p \lambda\right)$. Let $\mathscr{L}: \mathbb{B} \rightarrow L_{\#}^{p}(\Omega)$ with adjoint $\mathscr{L}^{*}$ and let $\vee$ denote the associated functional (4.15). If $1<p \lambda\left\|\mathscr{L}^{*} \varphi(f)\right\|_{\mathbb{B}^{*}}<\infty$, then $\mathbf{u}$ is a minimizer of (5.1) if and only if $\mathscr{L} \mathbf{u}$ and $\varphi(r)$ is an extremal pair and

$$
\left\|\mathscr{L}^{*} \varphi(r)\right\|_{\mathbb{B}^{*}}=\frac{1}{p \lambda}, \quad\left\langle\mathbf{u}, \mathscr{L}^{*} \varphi(r)\right\rangle=\frac{\|\mathbf{u}\|_{\mathbb{B}}}{p \lambda} .
$$


Proof. Since $\left\|\mathscr{L}^{*} \varphi(f)\right\|_{\mathbb{B}^{*}}>1 / p \lambda$ then $\|\mathbf{u}\|_{\mathbb{B}} \neq 0$ and we can now divide the equality on the right of (5.3) by $\|\mathbf{u}\|_{\mathbb{B}} \neq 0$ and (5.6) follows.

\section{REFERENCES}

[AV94] R. ACAR And C. R. Vogel, Analysis of Bounded Variation Penalty Methods of Ill-Posed Problems, Inverse Problems, 10 (1994), pp. 1217-1229.

[AF03] R. AdAms And J. Fournier, Sobolev Spaces, 2nd ed., Academic Press, 2003.

[Aji10] S. AJIEv, On Chebyshev centers, retractions, metric projections and homogeneous inverses for Besov, LizorkinTriebel, Sobolev and other Banach spaces, East J. of Approx.(2009), v.15(3), pp.375-428.

[ACM04] F. Andreu-VAillo, V. CASElles, AND J.M. Mazón, Parabolic Quasilinear Equations Minimizing Linear Growth Functionals, Birkhäuser Verlag, 2004.

[AXRNW13] P. Athavale., R. Xu, P. Radau., A. Nachman And G. Wright, Multiscale TV flow with applications to fast denoising and registration, in "SPIE Medical Imaging: image processing", Proc. of SPIE meeting, Lake Buena Vista, FL, Vol. 8669 86692K-1 (S. Ourselin, D. R. Haynor eds.) (2013).

[AV97] G. Aubert And L. Vese, A Variational Method in Image Recovery, SIAM J. Numer. Anal., 34 (1997), pp. 1948-1979.

[BCM05] A. Baudes, B. Coll And J.-M. Morel, A review of image denoising algorithms, with a new one, Multiscale Model. Simul. 4 (2005), no. 2, 490-530.

[BS88] C. Bennet And R. Sharpley, Interpolation of Operators, Academic Press, 1988.

[BB03] J. Bourgain AND H. BREZIS, On the equation $\operatorname{div} Y=f$ and application to control of phases, J. Amer. Math. Soc. 16 (2003), no. 2, 393-426.

[BB07] J. Bourgain And H. Brezis, New estimates for elliptic equations and Hodge type systems J. Eur. Math. Soc. (JEMS) 9 (2007), no. 2, 277-315.

[Bre10] H. BrezIS, Functional Analysis, Sobolev Spaces and Partial Differential Equations, Universitext, Springer, 2010.

[CL95] A. ChAmbolle AND P.L. LiOns, Image restoration by constrained total variation minimization and variants, SPIE Electronic Imaging Proceedings, Vol. 2567, 1995.

[CL97] A. Chambolle And P. L. LiOns, Image recovery via total variation minimization and related problems, Numer. Math., 76 (1997), pp. 167-188.

[CNV04] D. Cordero-Erauquin, B. NaZaret and C. Villani, A mass-transportation approach to sharp Sobolev and Gagliardo-Nirenberg inequalities, Adv. Math. 182 (2004), no. 2, 307-332.

[DFT05] B. Dacorogna, N. Fusco And L. TARTAR, On the solvability of the equation div $u=f$ in $L^{1}$ and in $C^{0}$, Rend. Mat. Acc. Lincei. s. 9 v. 14 (2003), pp. 239-245.

[DPD02] M. DEl PINo AND J. DolbeAult, Best constant for Gagliardo-Nirenberg inequalities and applications to nonlinear diffusions, J. Math. Pures Appl. 81 (2002), 847-875.

[DT84] F. Demengel And R. Temam, Convex functions of a measure and applications, Indiana Univ. Mathematics Journal 33(5): 673-709, 1984.

[DM87] R. DiPERnA AND A. MAJdA, Concentrations in regularizations for 2D incompressible flow, Comm. Pure and Appl. Math., 40 (1987), pp. 301-345.

[Ga57] E. GAGLIRADO, Caratterizzazioni delle tracce sulla frontiera relative ad alcuni classi di funzioni in $n$ variabili, Rend. Scm. Mat. Univ. Padova, 27 (1957) pp. 284-305.

[GLMV07] John B. Garnett, Triet M. Le, Yves Meyer And Luminita A. Vese, Image decompositions using bounded variation and generalized homogeneous Besov spaces, Appl. Comput. Harmon. Anal. 23 (2007), 25-56.

[HLTE10] M. Hidane, O. L'ezoray, V.-T. TA AND A. ElmoatAZ, Nonlocal multiscale hierarchical decomposition on graphs in "ECCV2010", Proc. 11th European Conf. Computer Vision, Heraklion, Crete, 2010, Vol. LNCS 6314 (Part IV) (K. Daniilidis, P. Maragos and N. Paragios eds.), Springer-Verlag (2010) pp. 638-650.

[HLE13] M. HidAne, O. LÉzoray AND A. Elmoataz, Nonlinear multilayered representation of graph-signals J. Math. Imaging and Vision 45(2) (2013) pp. 114-137.

[HRC10] M.HINTERMÜLLER AND M. RinCON-CAMACHO, Expected absolute value estimators for a spatially adapted regularization parameter choice rule in L1-TV-based image restoration, Inverse Problems 26(8) (2010).

[Jam47] R. C. JAMES Orthogonality and linear functionals in normed linear spaces, Trans. Amer. Math. Soc. 61 (1947), pp. 265-292.

[LV05] T. M. LE AND L. Vese, Image Decomposition Using Total Variation and div(BMO), Multiscale Modeling and Simulation, 4(2) (2005), 390-423. 
[LPSX06] D. Levy, D. Paquin, E. Schreibmann, And L. Xing, Multiscale Image Registration, Mathematical Biosciences and Engineering, 3, 2006, pp. 389-418.

[LNT00] M. Lopes Filho, H. J. NussenzVeig Lopes and E. Tadmor, Approximate solutions of the incompressible Euler equations with no concentrations, Annales de l'Institut Henri Poincare (C) Non Linear Analysis, 17 (2000) pp. 371-412.

[Ma06] Y. MADAY, $L^{\infty}$-stable approximation of a solution to $\operatorname{div}(\mathbf{Y})=f$ for $f \in L^{2}$ in two dimensions, J. Sci. Comput. 28(2-3) (2006), pp. 451-458.

[Ma07] V. MAZ'YA, Bourgain-Brezis type inequality with explicit constants, Interpolation theory and applications, 247252, Contemp. Math., 445, AMS Providence, RI, 2007.

[Me02] Y. MeYer, Oscillating Patterns in Image Processing and Nonlinear Evolution Equations, University Lecture Series Volume 22, AMS 2002.

[Mi10] P. Mironescu, On some inequalities of Bourgain, Brezis, Maz'ya, and Shaposhnikova related to $L^{1}$ vector fields, C. R. Math., 348 (910), 2010, pp. 513515.

[Mo84] V.A. Morozov, Methods for Solving Incorrectly Posed Problems, Springer Verlag, Heidelberg, 1984.

[Mo93] V.A. Morozov, Regularization Methods for Ill-Posed Problems, Florida, CRC Press, 1993.

[PL07] D. PaQuin, D. LeVy And L. XING, Hybrid Multiscale Landmark and Deformable Registration, Mathematical Biosciences and Engineering, 4 (2007), pp. 711-737.

[PT08] N.C. Phuc AND M. TORRES, Characterizations of the existence and removable singularities of divergencemeasure vector fields, Indiana Univ. Math. J. 57(4), 1573-1598, 2008.

[ROF92] L. Rudin, S. Osher AND E. FATEMI, Nonlinear Total Variation Based Noise Removal Algorithms, Physica D, 60, pp. 259-268, 1992.

[TNV04] E. TADMOR, S. NezZAR AND L. VeSe, A Multiscale Image Representation Using Hierarchical $(B V, L 2)$ Decomposition, Multiscale Modeling and Simulation: A SIAM Interdisciplinary Journal, Volume 2, Number 4, pp. 554-579, 2004.

[TNV08] E. TADMOR, S. NEZZAR AND L. VESE, hierarchical decomposition of images with applications to deblurring, denoising and segmentation, Communications in Math. Sciences 6(2) (2008) 281-307.

[TT11] E. TADMOR AND C. TAN, Hierarchical construction of bounded solutions of $\operatorname{div} U=F$ in critical regularity spaces, "Nonlinear Partial Differential Equations", Proc. 2010 Abel Symposium (H. Holden \& K. Karlsen eds.), Abel Symposia 7, Springer (2011), pp. 255-269.

[TH13] L.Tang And C. He, Multiscale Texture Extraction with Hierarchical $\left(B V, G p, L^{2}\right)$ Decomposition, J. Math. Imaging and Vision 45(2) (2013) 148-163.

[Tar94] L. TARTAR, Remarks on Some Interpolation Spaces, Center for Nonlinear Analysis, Research report 1-1-1994, Carnegie Mellon Univ.

[TA77] A. N. Tikhonov And V. A. Arsenin, Solution of Ill-posed Problems, Winston \& Sons, Washington, 1977.

[Ve92] I. VeCCHI, Gagliardo-Nirenberg imbeddings and DiPerna-Majda sequences, Proc. Roy. Soc. London Ser. A 439 (1992), no. 1906, pp. 337-340.

[VO04] L. VESE AND S. OSHER, Image denoising and decomposition with total variation minimization and oscillatory functions. Special issue on mathematics and image analysis. J. Math. Imaging Vision 20(1-2) (2004), pp. 7-18.

[Weyl46] H. WEYL The Princeton University Bicentennial Conference on the Problems of Mathematics, in "AMS History of Mathematics: A Century of Mathematics in America", Vol 2. Part II. (P. Duren et al., eds.), AMS, 1989.

[Woj91] P. WojtaszczYK, Banach Spaces for Analysts, Cambridge Univ. Press, 1991.

[Woj10] P. WOJTASZCZYK, private communication, 2010.

[Yos80] K. YosidA, Functional Analysis, 2nd ed., Springer, 1980.

Department of Mathematics, Center of Scientific Computation And Mathematical Modeling

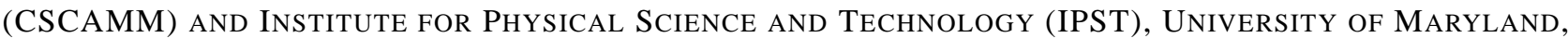
MD 20742.

E-mail address: tadmor@cscamm. umd.edu 